\title{
Aspectos centrales de la identificación y desarrollo de talentos deportivos: revisión sistemática Central aspects of the identification and development of sports talents: a systematic review
}

*Jairo Alejandro Fernandez Ortega, **Jeansy Alonso Rodriguez Buitrago, **Diego Alonso Sanchez Rodriguez

*Universidad Pedagógica Nacional de Colombia (Colombia), ${ }^{* *}$ Universidad de Ciencias Aplicadas y Ambientales (Colombia)

Resumen. La detección y el desarrollo de talentos deportivos ha sido durante décadas una estrategia utilizada a nivel internacional con el propósito de obtener deportistas de elite y para ello recluta desde edades muy tempranas a niños y niñas que son sometidos a elevados niveles de entrenamiento. Objetivo: el propósito de esta revisión fue identificar y sintetizar la literatura más importante sobre los efectos de la edad relativa (EER), el volumen de entrenamiento (VE), especialización temprana (ET) y diversificación de la práctica deportiva. (DPD) en el éxito deportivo. Metodología: Se realizaron búsquedas electrónicas en PubMed, ScienceDirect, SPORTDiscus (EBSCOhost), Science, Social Science, Web of Science, Scirus, Hinari, EMBASE, CINAHL, PsychINFO, Scopus y Ovid utilizando las palabras clave: edad relativa, especialización temprana, volumen de entrenamiento, diversificación deportiva. Resultados: Después de la selección contra los criterios establecidos se incluyeron 77 artículos fueron completamente revisados y contenían datos relevantes sobre EER, VE, ET y DPD. La calidad de la evidencia revisada fue buena. Conclusiones: La evidencia científica indica que el éxito en los deportistas de elite y súper elite, no está correlacionado con el VE, la ET, la EER. Estos hallazgos deben ser cuidadosamente considerados por todos aquellos involucrados en la identificación y desarrollo de los deportistas.

Palabras clave: Especialización temprana, entrenamiento intensivo, diversificación de deportes, jóvenes atletas, efecto de la edad relativa.

Abstract. The detection and development of sports talents has been for decades a strategy used internationally with the purpose of obtaining elite athletes, for this, it recruits boys and girls from a very early age who are subjected to high levels of training. Objective: The purpose of this review was to identify and synthesize the most important literature on the effects of relative age (ER), training volume (TV), early specialization (ES), and diversification of sports practice. (DSP) in sports success. Methodology: online search was made in PubMed, ScienceDirect, SPORTDiscus (EBSCOhost), Science, Social Science, Web of Science, Scirus, Hinari, EMBASE, CINAHL, PsychINFO, Scopus and Ovid using the keywords: relative age effect, early sport specialization, volume training, sports diversification. After selection based on the established criteria, 77 articles were included. They were completely reviewed and contained relevant data on ER, TV, ET, and DSP. The quality of the evidence reviewed was excellent in general terms. Conclusion: scientific evidence indicates that success in elite and super-elite athletes is not correlated with TV, ES, ER. These findings must be carefully considered by all those involved in the identification and development of athletes.

Key words: Early sport specialization, intensive training, sports diversification, young athletes, relative age effect.

\section{Introducción}

La lucha entre naciones para obtener medallas en los juegos Olimpicos y campeonatos del mundo se ha intensificado y condujo a que los paises incrementeran su inversión en los sistemas y estructuras deportivas para identificar y desarrollar atletas excepcionalmente talentosos, generando de esta forma un aumento significativo en el número de paises que logran medallas en dichos eventos. (De Bosscher, De Knop, Van Bottenburg, \& Shibli, 2006). El impacto del deporte en la sociedad provoco en los últimos 20 años un cambio en el propósito de las actividades deportivas que paso de ser recreativo, para convertirse en prácticas encaminadas hacía la obtención de altos niveles de desempeño deportivo. Las causas son multifactoriales e incluyen la importancia creciente que tiene el éxito deportivo en la sociedad, las recompensas financieras para los atletas y la percepción pública del valor de la competencia deportiva de élite (Feeley, Agel, \& LaPrade, 2016), aspectos que probablemente influyen en los atletas jóvenes para que tomen la decisión de iniciar un entrenamiento intensivo en un solo deporte desde edades muy tempranas.

Fecha recepción: 17-04-19. Fecha de aceptación: 15-07-20

Jairo Alejandro Fernandez Ortega

jairofdz@gmail.com
El relativo éxito obtenido en los países del este de Europa con la participación de deportistas muy jóvenes, contribuyó a crear la idea de la necesidad de mejorar los sistemas de selección, detección y a una iniciación temprana, en la práctica de un solo deporte. Idea que fue reforzada por entrenadores, investigadores y medios de comunicación emergiendo de esta forma como requisito para el éxito deportivo. (Abbott \& Collins, 2002) En países como la República Democrática Alemana, Unión Soviética, Bulgaria y Rumanía desde los años 60 y 70, se implementaron programas sistemáticos de especialización temprana (Bompa, 1994)

Dentro de estos procesos de detección y selección de talentos a edades tempranas se destacan cuatro aspectos centrales, la edad relativa, la especialización temprana, el volumen de entrenamiento y diversificación en el deporte.

La mayoría de programas de detección y selección de talentos clasifican a los niños sobre la base de la edad cronológica, lo cual indica que dentro de la misma categoría de edad podría existir una diferencia cronológica de hasta 12 meses, que puede ser mayor en términos de edad biológica, lo que genera diferencias -denominadas diferencias de edad relativa y que pueden conducir a resultados conocidos como efectos relativos de la edad (ERE) (Nakata, H. 2017a). Esto indica una asimetría en la distribución de la fecha de nacimiento que favorece a los participantes nacidos a comienzo del año de selección y discrimina a los nacidos a mediados y finales del año, e ignora las diferencias físicas, cognitivas y 
motivacionales (Lopez de Subijana, C., \& Lorenzo, J. 2018). Los niños relativamente mayores dentro de un grupo de edad, tienen más probabilidades de lograr el éxito deportivo, debido a que los niños relativamente mayores tienen ventajas en el crecimiento, madurez biológica y desarrollo cognitivo. (Campos,F. et al.2020) Además, tienen una mayor oportunidad de participar en competiciones y, en consecuencia, pueden mejorar sus habilidades psicológicas, técnicas y tácticas, apoyando así un mayor desarrollo atlético. (Romann \& Cobley, 2015)

El efecto relativo de la edad ha sido identificado en diversos deportes, como: Voleibol, (Campos, F.,Pellegrinotti I.,Campos, L., Dias, T., \& Gómez M.2020) Balonmano, (Rubia,A., Bjørndal, C., Sanchez, J,.Yague, J..Calvo, J., \& Maroto, S. 2020) Beisbol (Nakata, 2017 ), futbol (Sarmento, H., Anguera, M., Pereira, A., \& Araujo, D. 2018). Tenis (Edgar \& O’Donoghue, 2005), criquet (Connor, Renshaw, \& Doma, 2019; Jones, Lawrence, \& Hardy, 2018), baloncesto (Lopez de Subijana \& Lorenzo, 2018), rugby (Lemez, MacMahon, \& Weir 2016; McCarthy, Collins \& Court, 2016), Corredores de 60 metros (Romann \& Cobley, 2015), Esquiadores (Baker, Janning, Wong, Cobley, \& Schorer, 2014), Patinaje artístico (Baker, Janning, Wong, Cobley, \& Schorer, 2014) y múltiples deportes (Nakata \& Sakamoto,2011), en diversos países. Estos estudios identificaron una sobre representación de los participantes nacido en el primer cuartil del año. También se observó ERE significativo en la clasificación de los equipos en los torneos mundiales en todas las categorías.( Rubia, A. et al.2020) Aunque la mayoría de los estudios se han llevado a cabo en hombres los estudios realizados en mujeres no son tan concluyentes para identificar el efecto de ERE, en este sentido, se debe prestar especial atención ya que los resultados de los géneros masculino y femenino pueden ser contradictorios en todas las categorías (Campos,F. et al.2020)

Sin embargo este mejor rendimiento solo se presenta en las primeras etapas del desarrollo de los deportistas y no se mantiene a largo plazo (Henry, 2013) (Arrieta et al., 2016), porque los deportistas nacidos a principios de año pueden no cumplir con sus expectativas en el futuro cuando intentan alcanzar el nivel profesional.

La ERE genera un efecto negativo sobre las perspectivas de los atletas, debido a que los deportistas nacidos a finales del año se sienten presionados para desarrollar niveles de habilidad superiores a sus estadios de desarrollo y maduración para sobrevivir al sistema (Ford \& Willians 2012). Este efecto acumulativo de desventajas madurativas reducen la autoestima del niño y lo lleva a involucrarse menos que puede incluso conducir al abandono (Martin, Foels, Clanton, \& Moon, 2004). Por el contrario, los deportistas que maduran tardíamente tienen mayores probabilidades de desarrollar habilidades de autorregulación más adaptativas.(Cumming et al., 2018)

Otro aspecto importante asociado al desarrollo del talento deportivo es la idea que para lograr elevados niveles de desempeño son necesarios largos periodos de entrenamiento. Esto tiene una historia larga y controvertida. Estudios clásicos como los de, Simon \& Chase (1973) y Krogius (1976) desarrollados en jugadores de ajedrez observaron que se requerían 11.7 años de preparación para alcanzar el nivel de maestro internacional de ajedrez. Esta «regla de los 10 años»» de Simon and Chase (1973) está respaldada por datos de una amplia gama de estudios en diversos escenarios: música (Sosniak, 1985), matemáticas (Gustin, 1985), tenis (Monsaas, 1985), natación (Kalinowski, 1985) y carreras de larga distancia (Wallingford, 1975). Sin embargo, la relación entre el rendimiento adquirido y la cantidad de práctica y experiencia resultó ser débil o moderada (Ericsson, Krampe, \& TeschRomer 1993) (W. Helsen, J. Starkes, \& N. Hodges, 1998; Starkes, 2000) y la calidad de la evidencia que soporta la aplicabilidad de la regla de 10 años / 10.000 h, está limitada por la calidad moderada de los estudios. (Rees et al.2016). Los volúmenes de entrenamiento pueden diferir entre los diferentes sistemas deportivos (Ford et al., 2012; Güllich y Emrich, 2013) y los tipos de deportes. Por ejemplo, los mejores atletas en deportes de resistencia (Deakin y Cobley, 2003; Law, Côté y Ericsson, 2008; Seiler y Tonnessen, 2009) generalmente tienden a realizar más práctica /entrenamiento que los deportes de equipo (Baker, Cote, \& Abernethy, 2003; Memmert, Baker, \& Bertsch, 2010; Van Rossum, 2000).

Guillich (2014) en un estudio en jugadores de hockey observo que los Campeones Olímpicos acumularon $2183 \pm$ 1388 horas de práctica / entrenamiento deportivo específico organizado antes de obtener su primera medalla a nivel nacional y $4393 \pm 1389$ horas antes de obtener su primera medalla en campeonatos internacionales de alto nivel. Estos resultados son consistentes con otras investigaciones en diversos deportes (Carlson, 1988; Johnson, 2006; Ronbeck, Dunnagan y Stewart, 2005; Van Rossum, 2000) que ratifican que los deportistas de clase mundial no acumulan más práctica / entrenamiento específico para el deporte, sino que participa más ampliamente en varios deportes.

Se ha sugerido que la especialización temprana es un requisito en los deportes donde el rendimiento máximo ocurre antes de la madurez (p. Ej., Patinaje artístico femenino, gimnasia rítmica), lo que ha generado una creciente preocupación y controversia sobre los efectos que tiene la especialización deportiva a temprana edad. La especialización deportiva se caracteriza por el entrenamiento durante todo el año en un solo deporte, con exclusión de otros deportes o actividades no deportivas.

El apoyo explícito a la especialización es escaso y varias federaciones de medicina deportiva y ciencias del ejercicio desde la década de los noventa han desalentado su práctica. La Federación Internacional de Medicina del Deporte (1991), la Federación de Medicina del Deporte de Nueva Zelanda (Gerrard 1993), la Federación Europea de Psicología del Deporte (1996) y la Organización Mundial de la Salud (1997) coinciden en resaltar que la especialización temprana y el tipo de entrenamiento a que ella conlleva, no tiene justificación fisiológica, psicológica o educativa. La Academia Estadounidense de Pediatría (1991) propuso que se permitiera a los niños practicar una variedad de deportes que alentara el juego libre no estructurado para mejorar el disfrute de los deportes, así como promover la espontaneidad y la creatividad.

Existe suficiente evidencia que señala que la especialización antes de la pubertad es perjudicial (Jayanthi, 2013) y algunos autores equiparan este tipo de entrenamiento con el abuso infantil (Rowland, 1997). Por otra parte varios estu- 
dios retrospectivos indican que la especialización tardía (definido como mayores de 12 años de edad) en un deporte en particular, puede generar mejores resultados en los logros deportivos de elite que la especialización temprana. Los datos disponibles entre las poblaciones de atletas indican que menos del $1 \%$ de los que se especializaron en un deporte a temprana edad alcanzaron el nivel elite o profesional (Myer, 2016; Mostafavifar, 2013; Malina, 2010; Guellich, 2006; Latorre, Garcia, \& Lopez, 2018).

Varios estudios han demostrado que el éxito competitivo a nivel juvenil e infantil no se correlaciona (o modestamente en el mejor de los casos) con el éxito a nivel sénior (Vaeyens, 2009; Gullich, 2014; Barreiros, 2014; Brouwers, 2012), lo que ratifica el postulado que no es fiable predecir un nivel rendimiento a la edad adulta a partir de una debido a que sólo es posible predecir un nivel de rendimiento cuando el deportista está cerca de la edad de su madurez (Martindale, R. J., Collins, D., \& Daubney, J. 2005)

Ahora bien, desde una perspectiva epidemiológica existe preocupación por los efectos que sobre la salud tiene la especialización temprana. La evidencia científica indica que la especialización deportiva temprana es un factor de riesgo que pude generar varios tipos de lesión que abarcan las diferentes esferas del desarrollo de los niños: aislamiento social, sobredependencia, síndrome de Burnout, manipulación, lesiones y compromisos en los procesos de desarrollo y maduración. (Bush, C., Wilhelm,A., Lavallee, M.,\& Deitch, J., 2019; Malina 2010),

Brenner y colaboradores (Brenner, 2016) en un informe clínico retoma y complementa dos documentos de la Academia Norte Americana de Pediatría (AAP): El entrenamiento intensivo y especialización deportiva en atletas jóvenes (2000) y las Lesiones por sobreuso, sobreentrenamiento y agotamiento en atletas niños y adolescentes (2007), destaca las preocupaciones fisiológicas específicas de la especialización deportiva en atletas.

Sin embargo, a pesar de toda esta evidencia las tasas de especialización deportiva a nivel mundial son elevadas y continúan incrementándose dramáticamente.

En contraposición a el concepto de especialización temprana surge la propuesta de la práctica de múltiples deportes, que además de reducir la práctica especifica en un deporte tiene el potencial de generar efectos positivos a nivel físico, cognitivo, psicológico, social (Malina, 2010; Jayanthi, 2013; Côté, 2009; Moesch, 2011) y procesos de adquisición y refinación de habilidades específicas (Vaeyens, Güllich, Warr y Philippaerts, 2009; Gullich, A., Kovar, P., Zart, S., \& Reimann, A. 2017).

Las experiencias tempranas de aprendizaje en diversos deportes generan varios efectos positivos en los jóvenes atletas: a) mejoran la eficacia de la práctica en el deporte específico, obteniendo más rápidamente un buen nivel de rendimiento invirtiendo menor tiempo en la práctica del deporte especifico (Carlson, 1988; Gullich, 2014; Hornig, 2016; Gullich, 2014; Gullich, 2017); b) facilitan los procesos de adquisición y afinamiento de habilidades específicas (Vaeyens, 2009); los atletas adquieren un repertorio multifacético en términos de una red más amplia de habilidades motoras perceptivas que favorecen respuestas funcionales las cuales permiten a los jóvenes resolver de forma eficiente situa- ciones motoras inmediatas (Ford, 2012; Wolpert, 2011); c) la experiencia en varios deportes aumenta la probabilidad de que un atleta elija el deporte en el que tiene más talento (Gullich, 2014). El entrenamiento físico diversificado -es decir, el ejercicio multiactividad no especializado- puede tener efectos positivos en el funcionamiento de los sistemas endocrino, muscular, nervioso y cardiovascular (American Academy of Pediatrics, 2000).

El proposito del presente estudio es realizar una revisión sistemática basada en evidencia, con el objetivo de determinar la solidez de los diversos postulados en torno al entrenaiento intrensivo precoz y a la especilización temprana.

\section{Método}

Se realizó una revisión sistemática de la literatura disponible en las bases de datos Pubmed, Proquest, SPORTDiscus, (EBSCOhost), Science, Social Science, Web of Science (ISI), Scirus, Hinari, EMBASE, CINAHL, PsychINFO, PEDro Scopus y Ovid, siguiendo la guía PRISMA (Moher et al., 2015) (Preferred Reporting Items for Systematic Reviews and Meta-analyses) se utilizaron las palabras clave: edad relativa, especialización temprana, volumen de entrenamiento y diversificación de la práctica deportiva. Cada palabra se combinó con los términos: talento deportivo, experto, atleta de elite, detección. Solo se incluyeron artículos retrospectivos metanalisis o revisiones.

Para la selección primaria de los artículos se establecieron los siguientes criterios de inclusión: Estudios retrospectivos que analizaran los efectos de la edad relativa (ER), el volumen de entrenamiento, la especialización temprana y la diversificación de la práctica deportiva; Artículos publicados en revistas, indexadas que se encuentren dentro de SCIMAGO (International Scientific Journal \& Country Ranking) realizados en cualquier tipo de deporte y publicados entre 1980 a junio de 2019. No hubo restricción de idioma durante las búsquedas, se excluyeron los estudios descriptivos o que no fueron realizados en deportistas de elite, o que no contenían información relevante sobre los cuatro criterios del desarrollo del talento deportivo.

Las listas fueron combinadas usando el gestor bibliográfico EndNoteTM X7 y los duplicados fueron suprimidos, al igual que los estudios que no estaban disponibles como publicaciones completas. De este listado dos revisores seleccionaron de forma independiente los resúmenes para identificar los artículos que potencialmente cumplían con los criterios de inclusión. De esos artículos se recuperaba la versión de texto completo que era revisada de forma independiente por cada uno de los expertos para determinar si cumplían con los criterios de inclusión. En caso de existir desacuerdo con respecto a la elegibilidad del estudio, un tercer experto revisaba el estudio.

La búsqueda bibliográfica se realizó entre los meses de enero y agosto de 2019 obteniendo un total de 468 artículos y se identificaron 25 artículos adicionales mediante referencias cruzadas. El proceso se repitió en el mes de junio de 2020 para identificar cualquier artículo nuevo publicado desde la búsqueda inicial. 


\section{Control de calidad de los artículos}

La calidad metodológica para los estudios cualitativos se evaluó utilizando los formularios de revisión crítica propuestos por Letts, Wilkins, Stewart, Bosch, \& Westmorland, (2007) que evalúa 21 aspectos y para los estudios cuantitativos la guía de Law, Stewart, \&Pollock (1998.) que contempla 16 aspectos.

En los artículos cualitativos se verifico si cumplían con los 21 aspectos determinados en la guía: objetivo, literatura revisada, diseño del estudio (tres aspectos), muestreo (cuatro aspectos), recolección de datos (cuatro aspectos), análisis de datos (cinco aspectos), rigor general (un aspecto) y conclusión / implicaciones (dos aspectos).

Para los estudios cuantitativos se verifico si incluían los 16 aspectos definidos en la guía: objetivo, relevancia de la literatura, muestra (dos aspectos), procedimiento de consentimiento informado, medidas de resultado, validez de las medidas, importancia de los resultados, análisis, importancia clínica, descripción de los abandonos, conclusión, implicaciones prácticas y limitaciones. Para el presente estudio los aspectos de importancia clínica y procedimiento de intervención no se aplicaron porque los estudios no incluían intervenciones.

Para valor el cumplimiento de cada uno de estos aspectos se creó una matriz de análisis donde se asignaba uno (1) cuando cumplía con los criterios, o cero cuando no cumplía con los criterios completamente, o NA (no aplica). Se calculó el puntaje final expresado como un porcentaje para cada estudio siguiendo las pautas de puntaje de Faber, Bustin, Oosterveld, Elferink-Gemser, \& Nijhuis-Van der Sanden, (2016) y Wierike, Vander Sluis, Vanden Akker-Scheek, ElferinkGemser, \& Visscher, (2013).

La puntuación final de cada artículo se obtenía de la sumatoria del valor asignado en cada aspecto, que se dividido por el número total de aspectos puntuados según el de investigación específico (es decir, 16 o 21). En el presente estudio se adoptaron las clasificaciones propuestas por Faber et al. (2016) y Wierike et al., (2013) que clasifican la calidad de los artículos de la siguiente forma; a) baja calidad metodológica, con un puntaje de d»50\%; b) buena calidad metodológica: puntaje entre 51 y $75 \%$; excelente calidad metodológica, con una puntuación d»75\%. Se utilizó la plantilla de extracción de datos de Cochrane (Cochrane Consumers and Communication Review Group, 2016), adaptado a los requisitos de inclusión de estudios de esta revisión, posteriormente se realizó una prueba piloto con 15 artículos seleccionados al azar. Un revisor extrajo los datos y otro realizo la verificación.

\section{Resultados}

La búsqueda inicial identificó un total de 557 documentos que fueron exportados al gestor bibliográfico (EndNote TM X7 Clarivate Analytics, Philadelphia, PA, USA) y 289 duplicados fueron eliminados. Los 268 restantes se revisaron a partir del título y el resumen y se excluyeron 143 y los 125 restantes fueron analizados a partir del texto completo de los cuales 85 se eliminaron por no cumplir con los criterios de inclusión, finalmente se analizaron en profundidad 40 estudios. (Fig.1) La razón principal para la exclusión del estudio $(\mathrm{n}=53)$ fue falta de relevancia para el tema de investigación de esta revisión o la inclusión de deportistas amateurs $(\mathrm{n}=$ 32).

El análisis cronológico de los artículos considerados en esta revisión, indica que el 55,1\% de los estudios fue publicado entre 2010 y 2019.

Comprender o explicar el rendimiento deportivo en los atletas de elite implica analizar a la luz de la evidencia científica, varios aspectos que durante muchas décadas han sido catalogados como primordiales para llegar a los máximos niveles de experticia en el deporte; los efectos de la edad relativa, la especialización temprana, el volumen de entrenamiento y la diversificación en la práctica deportiva, que si bien es cierto que se abordaran de forma independiente, se encuentran altamente relacionados. Sobre la base de esta lógica teórica los resultados se presentaran desde cada una de estas categorías.

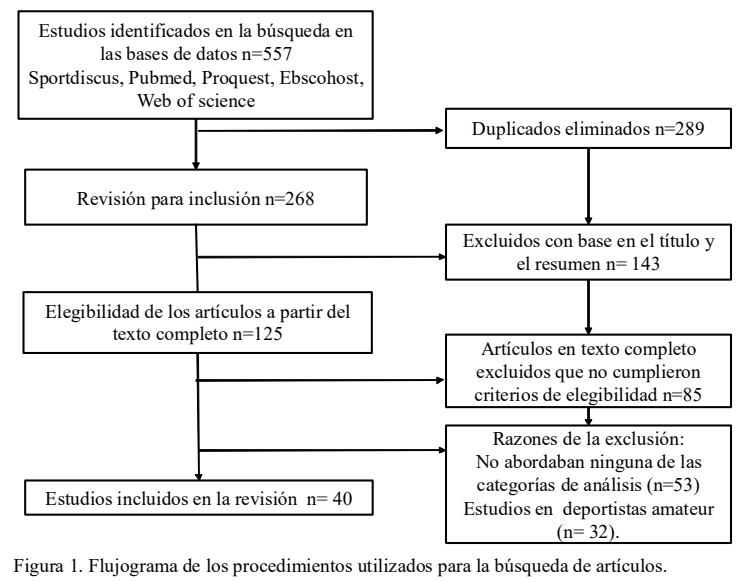

\section{Efectos de la edad relativa}

La mayoría de programas de detección y selección de talentos a nivel mundial en todos los deportes, clasifican los niños sobre la base de la edad cronológica, aspecto que es insensible a las sutiles diferencias que se presentan entre los miembros de una cohorte de edad y está asociado a consecuencias importantes para el desarrollo de los atletas, conocidas como «efectos relativos de la edad» (ERE) ( Baker \& Logan, 2007) (Cobley, Baker, Wattie, \& McKenna, 2009)

La presente revisión identificó un consenso en la literatura sobre la sobrerrepresentación de los deportistas nacidos en los cuartiles 1-2 del año en diversos deportes y en varios países. (Tabla 1) Los estudios analizados presentaron una correlación significativa ( $\mathrm{p}<0,001)$ entre el ERE y el éxito definido por las clasificaciones finales de los equipos juveniles. Los análisis de regresión revelaron que los equipos con una mediana de fechas de nacimiento en el primer trimestre del año, terminan mejor clasificados.(Augste \& Lames, 2011) Solo en el caso del rugby fenemenino en la primera fase, no se identificaron efectos de la ERE, pero se explica por que se trataba en ese momento de un deporte donde se iniciaban las mujeres. El conjunto de estudios muestran que la identificación del talento puede verse significativamente afectada por ERE.

\section{Volumen de entrenamiento}

Otro de los postulados que ha sido altamente discutido y centro de gran controversia ha sido el VE que se requiere 
Tabla 1

\begin{tabular}{|c|c|c|c|c|c|c|c|c|}
\hline Estudio & Deporte & $\begin{array}{l}\text { Tipo de } \\
\text { estudio }\end{array}$ & Población & Sexo & Edad & $\begin{array}{l}\text { Nivel } \\
\text { competitivo }\end{array}$ & Instrumentos & Principales resultados \\
\hline Nakata, 2017 & Beisbol & $\begin{array}{l}\text { Análisis } \\
\text { retrospectivo }\end{array}$ & $\mathrm{n}=4218$ & Hombres & NR & Profesionales & $\begin{array}{l}\text { Las fechas de nacimiento de los jugadores y } \\
\text { la duración de las carreras profesionales se } \\
\text { obtuvieron de una publicación oficial. }\end{array}$ & $\begin{array}{l}\text { El número de jugadores con carreras profesionales de más de } \\
19 \text { años fue significativamente menor en Q4 que en Q1, Q2 y } \\
\text { yQ3. El efecto relativo de la edad se asoció con la duración de } \\
\text { las carreras profesionales. La edad relativa parece ser un factor } \\
\text { muy importante para el desarrollo de la experiencia entre los } \\
\text { jugadores analizados e implica desventajas a largo plazo } \\
\text { después de convertirse en jugadores profesionales. }\end{array}$ \\
\hline $\begin{array}{l}\text { Edgar \& } \\
\text { O'Donoghue, } 2005\end{array}$ & Tenis & $\begin{array}{l}\text { Análisis } \\
\text { retrospectivo }\end{array}$ & $\mathrm{n}=924$ & $\begin{array}{l}\text { Hombres y } \\
\text { mujeres }\end{array}$ & $\mathrm{NR}$ & $\begin{array}{l}\text { Elite sénior } \\
\text { junior }\end{array}$ & $\begin{array}{l}\text { La información y general y las fechas de } \\
\text { nacimiento se obtuvieron de la paginas } \\
\text { ofíciales de los torneos de gran slam 2002- } \\
2003\end{array}$ & $\begin{array}{l}\text { Identifico un efecto significativo de la EER }(\mathrm{P}<0.001) \text {, donde } \\
\text { el } 58.9 \% \text { de los tenistas elite sénior y el } 59.5 \% \text { de los elite } \\
\text { júnior, nacieron en los primeros } 6 \text { meses del año }\end{array}$ \\
\hline Connor, et al. 2019 & Críquet & $\begin{array}{l}\text { Análisis } \\
\text { retrospectivo } \\
(2011-2015)\end{array}$ & $\mathrm{n}=2415$ & $\begin{array}{l}\text { Hombres y } \\
\text { mujeres }\end{array}$ & $15-19$ & Junior y Sénior & $\begin{array}{l}\text { La información se obtuvo de una base de } \\
\text { datos en línea de código abierto } \\
\text { (nationalchamps.com.au), y la organización } \\
\text { nacional (Cricket Australia) proporcionó } \\
\text { acceso a la fecha de nacimiento, habilidad } \\
\text { dominante y capacidad de entrega. }\end{array}$ & $\begin{array}{l}\text { Los hallazgos de este estudio resaltan la influencia de los } \\
\text { nefectos relativos de la edad tanto para los jugadores de cricket } \\
\text { junior masculino como femenino. El } 56 \% \text { nacieron en los } \\
\text { primeros meses del año }\end{array}$ \\
\hline $\begin{array}{l}\text { Lopez de Subijana \& } \\
\text { Lorenzo, } 2018\end{array}$ & $\begin{array}{l}\text { Baloncesto, } \\
\text { Futbol }\end{array}$ & $\begin{array}{l}\text { Análisis } \\
\text { retrospectivo } \\
2004-2013\end{array}$ & $\mathrm{n}=1224$ & Hombres & NR & $\begin{array}{l}\text { Profesional } \\
\text { elite junior }\end{array}$ & $\begin{array}{l}\text { La información y general y las fechas de } \\
\text { nacimiento se obtuvieron de las páginas } \\
\text { oficiales de la primera liga de futbol y de } \\
\text { baloncesto, de los campeonatos oficiales } \\
\text { europeos y de las federaciones nacionales. }\end{array}$ & $\begin{array}{l}\text { 42.3\% de los jugadores junior nacieron con mayor frecuencia } \\
\text { en el primer trimestre del año que los jugadores profesionales } \\
(30.7 \%) \mathrm{p}<0.001 \text { y Por las fechas de nacimiento del cuarto } \\
\text { trimestre fueron más frecuentes entre los jugadores } \\
\text { profesionales }(18.0 \%) \text { que entre los jugadores de nivel junior } \\
(9.2 \%) \text {. }\end{array}$ \\
\hline Lemez, et al.2016 & Rugby & $\begin{array}{l}\text { Análisis } \\
\text { retrospectivo } \\
2006-2010\end{array}$ & $\mathrm{n}=15984$ & Mujeres & NR & Elite & $\begin{array}{l}\text { La información y general y las fechas de } \\
\text { nacimiento se obtuvieron de la paginas } \\
\text { oficiales de la Unión Mundial de Rugby; } \\
\text { Rugby Canadá y la Unión de Rugby de } \\
\text { Nueva Zelanda }\end{array}$ & $\begin{array}{l}\text { No encontraron evidencias de un EER en las muestras de la } \\
\text { Copa del Mundo, pero los análisis de chi cuadrado } \\
\text { identificaron algunos patrones típicos y atípicos de EER en los } \\
\text { niveles de desarrollo. Los grupos de deportistas de los últimos } \\
\text { años mostraron un patrón de EER típico con una mayor } \\
\text { representación de jugadores mayores, mientras que los grupos } \\
\text { más antiguos mostraron un patrón atípico de EER con una } \\
\text { mayor representación de jugadores más jóvenes }\end{array}$ \\
\hline $\begin{array}{l}\text { Nakata \& } \\
\text { Sakamoto,2011 }\end{array}$ & $\begin{array}{l}\text { Varios } \\
\text { deportes }\end{array}$ & $\begin{array}{l}\text { Análisis } \\
\text { retrospectivo }\end{array}$ & $\mathrm{n}=4318$ & Hombres & NR & Profesionales & $\begin{array}{l}\text { La información y general y las fechas de } \\
\text { nacimiento se obtuvieron de las páginas } \\
\text { oficiales de las ligas nacionales de futbol, } \\
\text { baloncesto, beisbol, voleibol, balonmano, } \\
\text { rugby, futbol americano y sumo. }\end{array}$ & $\begin{array}{l}\text { Se observó un efecto significativo de la edad relativa en } \\
\text { béisbol, fútbol, voleibol, Ekiden, baloncesto, sumo y } \\
\text { equitación. Y no significativa en balonmano, rugby y } \\
\text { bádminton, aunque el año escolar en Japón comienza el } 1 \text { de } \\
\text { abril, se observan efectos significativos relativos a la edad en } \\
\text { algunos eventos deportivos. }\end{array}$ \\
\hline Baker, et al , 2014 & Esquiadores & $\begin{array}{l}\text { Análisis } \\
\text { retrospectivo }\end{array}$ & $\mathrm{n}=19432$ & $\begin{array}{l}\text { Hombres y } \\
\text { mujeres }\end{array}$ & $\mathrm{NR}$ & Elite sénior & $\begin{array}{l}\text { La información y general y las fechas de } \\
\text { nacimiento se obtuvieron de las páginas } \\
\text { oficiales de la federación internacional de } \\
\text { Sky. }\end{array}$ & $\begin{array}{l}\text { Los análisis de chi-cuadrado revelaron un efecto significativo } \\
\text { de la ERE en la mayoría de las modalidades y entre géneros }\end{array}$ \\
\hline Baker, et al. 2014 & $\begin{array}{l}\text { patinaje } \\
\text { artistico, } \\
\text { gimnasia }\end{array}$ & $\begin{array}{l}\text { Análisis } \\
\text { retrospectivo } \\
1997-2007\end{array}$ & $\mathrm{n}=1114$ & $\begin{array}{l}\text { Hombres y } \\
\text { mujeres }\end{array}$ & $\begin{array}{l}22,6- \\
14,6\end{array}$ & $\begin{array}{l}\text { Elite sénior y } \\
\text { junior }\end{array}$ & $\begin{array}{l}\text { La información y general y las fechas de } \\
\text { nacimiento se obtuvieron de los archivos } \\
\text { oficiales de las federaciones Canadienses } \\
\text { de Patinaje y gimnasia }\end{array}$ & $\begin{array}{l}\text { No se observó ERE para los patinadores, pero si atípico para } \\
\text { los gimnastas. }\end{array}$ \\
\hline McCarthy, et al. 2016 & $\begin{array}{l}\text { Rugby, } \\
\text { Criquet }\end{array}$ & $\begin{array}{l}\text { Análisis } \\
\text { retrospectivo }\end{array}$ & $\mathrm{n}=1489$ & Hombres & NR & $\begin{array}{l}\text { Profesional y } \\
\text { elite }\end{array}$ & $\begin{array}{l}\text { La información y general y las fechas de } \\
\text { nacimiento se obtuvieron de los archivos } \\
\text { oficiales de Unión de Rugby y de Criquet } \\
\text { de Inglaterra. }\end{array}$ & $\begin{array}{l}\text { Observaron una sobrerrepresentación significativa de los } \\
\text { jugadores nacidos en los primeros meses del año en la } \\
\text { selección inicial en el rugby y el cricket. }\end{array}$ \\
\hline Campos et al. 2020 & Voleibol & $\begin{array}{l}\text { Análisis } \\
\text { retrospectivo } \\
2007-2017\end{array}$ & $\mathrm{n}=1654$ & Mujeres & NR & Elite & $\begin{array}{l}\text { La información y general y las fechas de } \\
\text { nacimiento se obtuvieron de los archivos } \\
\text { oficiales de la federación internacional de } \\
\text { Voleibol }\end{array}$ & $\begin{array}{l}\text { En general, el } 61.3 \% \text { de los atletas nacieron en Q1, el } 29,1 \% \text { en } \\
\text { Q2 y el } 9,6 \% \text { entre Q3 y Q4. Se identificó un efecto } \\
\text { significativo }<<0,01\end{array}$ \\
\hline Rubia et al. 2020 & Balonmano & $\begin{array}{l}\text { Análisis } \\
\text { retrospectivo } \\
\text { 2013-2018 }\end{array}$ & $\mathrm{n}=6631$ & $\begin{array}{l}\text { Hombres y } \\
\text { mujeres }\end{array}$ & $\mathrm{NR}$ & $\begin{array}{l}\text { Elite Sénior y } \\
\text { Junior }\end{array}$ & $\begin{array}{l}\text { La información y general y las fechas de } \\
\text { nacimiento se obtuvieron de los archivos } \\
\text { oficiales de la federación internacional de } \\
\text { Balonmano }\end{array}$ & $\begin{array}{l}\text { Se observó ERE significativo en la clasificación de los equipos } \\
\text { en los torneos mundiales en todas las categorías y en la } \\
\text { selección de los jugadores hombres y mujeres. La persistencia } \\
\text { de la RAE en el balonmano está lejos de ser sorprendente } \\
\text { debido a la alta correlación que se ha observado entre el nivel } \\
\text { de rendimiento del atleta y las demandas físicas y fisiológicas. }\end{array}$ \\
\hline Jones, et al., 2018 & Críquet & $\begin{array}{l}\text { Análisis } \\
\text { retrospectivo }\end{array}$ & $\mathrm{n}=262$ & Hombres & NR & Elite Sénior & $\begin{array}{l}\text { La información y general y las fechas de } \\
\text { nacimiento se obtuvieron de los archivos } \\
\text { oficiales de la federación internacional de } \\
\text { cricket }\end{array}$ & $\begin{array}{l}\text { Las frecuencias de distribución para todas las posiciones reveló } \\
\text { que Q1 estaba significativamente sobrerrepresentado }(\mathrm{p}<0.01) \\
\text { mientras que Q4 estaba subrepresentado significativamente (p } \\
<0.01) \text {. }\end{array}$ \\
\hline $\begin{array}{l}\text { Rubajczyk, \& Rokita } \\
2017\end{array}$ & Baloncesto & $\begin{array}{l}\text { Análisis } \\
\text { retrospectivo, } \\
\text { 2013-2016 }\end{array}$ & $\mathrm{n}=7268$ & $\begin{array}{l}\text { Hombres y } \\
\text { mujeres }\end{array}$ & $\mathrm{NR}$ & $\begin{array}{l}\text { Elite sénior y } \\
\text { junior }\end{array}$ & $\begin{array}{l}\text { La información y general y las fechas de } \\
\text { nacimiento se obtuvieron de los archivos } \\
\text { oficiales de la federación Polaca de } \\
\text { baloncesto }\end{array}$ & $\begin{array}{l}\text { El estudio observo tanto en hombre como mujeres y en las dos } \\
\text { categorías una sobre representación de Q1 y una } \\
\text { subrepresentación del Q4 }(\mathrm{p}<0.001)\end{array}$ \\
\hline Arrieta, et al.2015, & Baloncesto & $\begin{array}{l}\text { Análisis } \\
\text { retrospectivo }\end{array}$ & $\mathrm{n}=2395$ & $\begin{array}{l}\text { Hombres y } \\
\text { mujeres }\end{array}$ & $\mathrm{NR}$ & $\begin{array}{l}\text { Elite Junior, } \\
\text { sénior }\end{array}$ & $\begin{array}{l}\text { La información y general y las fechas de } \\
\text { nacimiento se obtuvieron de los archivos } \\
\text { oficiales de la federación internacional de } \\
\text { baloncesto }\end{array}$ & $\begin{array}{l}\text { El estudio observo tanto en hombre como mujeres y en las dos } \\
\text { categorías una sobre representación de Q1 y una } \\
\text { subrepresentación del Q4 ( } \mathrm{p}<0.001)\end{array}$ \\
\hline Brustio, et al. 2019 & Atletismo & $\begin{array}{l}\text { Análisis } \\
\text { retrospectivo } \\
2007,2018\end{array}$ & $\mathrm{n}=39590$ & $\begin{array}{l}\text { Hombres y } \\
\text { mujeres }\end{array}$ & NR & $\begin{array}{l}\text { Elite Junior, } \\
\text { Sénior }\end{array}$ & $\begin{array}{l}\text { La información y general y las fechas de } \\
\text { nacimiento se obtuvieron de los archivos } \\
\text { oficiales de la federación internacional de } \\
\text { atletismo }\end{array}$ & $\begin{array}{l}\text { Independientemente del género, los resultados sugirieron que } \\
\text { la probabilidad de ser incluido en el rango de los } 100 \text { mejores } \\
\text { es mayor para un atleta nacido en el Q1 que uno nacido en el } \\
\text { Q4, Además, los RAE son probablemente más fuertes en los } \\
\text { hombres en comparación con las mujeres en todas las } \\
\text { categorías. }\end{array}$ \\
\hline Ferriz, et al. 2020 & Triatletas & $\begin{array}{l}\text { Análisis } \\
\text { retrospectivo }\end{array}$ & $\mathrm{n}=160$ & $\begin{array}{l}\text { Hombres y } \\
\text { mujeres }\end{array}$ & $15-17$ & Elite Junior & $\begin{array}{l}\text { La información y general, fechas de } \\
\text { nacimiento y rendimiento se obtuvieron de } \\
\text { los archivos oficiales de la federación } \\
\text { Valenciana de Triatlón }\end{array}$ & $\begin{array}{l}\text { Hay mayor selección de deportistas nacidos en Q1, } \\
\text { significativamente diferente }(\mathrm{p}<0.001) \text { de Q3 y Q4, tanto en } \\
\text { hombres como en mujeres. }\end{array}$ \\
\hline
\end{tabular}

para llegar a los altos logros deportivos. La idea que acumular una gran cantidad de entrenamiento y competencia conlleva al máximo rendimiento y al éxito deportivo, tiene un historial extenso y controvertido que podría tener sus inicios en el siglo 18 (Ericsson, Krampe, \& Tesch-Romer 1993)

La propuesta del volumen ideal de entrenamiento para llegar al alto rendimiento surge de los trabajos de Simon \& Chase, (1973) que fueron retomados por Ericsson, Krampe, \&Tesch-Romer (1993) y ratificada en el estudio de MacMahon, Helsen, Starkes, \& Weston, (2007) que plantean la famosa regla que se requieren 10000 horas de trabajo específico para lograr el éxito deportivo. Sin embargo la evidencia identificada demuestra que no es necesaria esa cantidad de VE (Tabla2)

Desde el punto de vista de las lesiones se observó que los atletas que realizaron altos volúmenes de entrenamiento en el deporte específico, fueron los que presentaron un nivel más elevado de lesiones por uso excesivo.(Post et al., 2017)

\section{Especialización temprana}

Este fenómeno está ligado al concepto anterior, donde la necesidad de acumular grandes volúmenes de entrenamien- 
Tabla 2

\begin{tabular}{|c|c|c|c|c|c|c|c|c|}
\hline \multicolumn{9}{|c|}{ Volumen de entrenamiento } \\
\hline Estudio & Deporte & $\begin{array}{l}\text { Tipo de } \\
\text { estudio }\end{array}$ & Población & Sexo & Edad & $\begin{array}{l}\text { Nivel } \\
\text { d competitivo }\end{array}$ & Instrumentos & Principales resultados \\
\hline Gullich, 2014 & Hockey & $\begin{array}{l}\text { Análisis } \\
\text { retrospectivo }\end{array}$ & $\mathrm{n}=16$ & Hombres & 26,6 & Elite & $\begin{array}{l}\text { Cuestionario estandarizado administrado } \\
\text { por email y de forma personal }\end{array}$ & $\begin{array}{l}\text { Los Campeones Olímpicos realizaron volúmenes moderados de } \\
\text { entrenamiento y obtuvieron su primera medalla internacional } \\
\text { de alto nivel después de acumular } 4393 \pm 1389 \text { pero se } \\
\text { dedicaron a otras actividades deportivas extensas durante la } \\
\text { infancia y la juventud. }\end{array}$ \\
\hline Hornig, et al.2016 & Futbol & $\begin{array}{l}\text { Estudio } \\
\text { retrospectivo }\end{array}$ & $\mathrm{n}=52$ & Hombres & NR & Profesionales & $\begin{array}{l}\text { Cuestionario estandarizado administrado } \\
\text { por email y de forma personal }\end{array}$ & $\begin{array}{l}\text { Los jugadores acumularon en promedio } 4264 \text { horas durante } ~ \\
16 \text { años antes de debutar en la } 1^{\text {a }} \text { división de la Bundesliga. El } \\
\text { debut en el equipo nacional estuvo precedido por } 4532 \text { horas } \\
\text { (promedio) durante } \sim 17 \text { años }\end{array}$ \\
\hline Baker, et al, 2003 & $\begin{array}{l}\text { Hockey } \\
\text { Baloncesto y } \\
\text { Netball }\end{array}$ & $\begin{array}{l}\text { Estudio } \\
\text { retrospectivo } \\
\text { Correlacional }\end{array}$ & $\mathrm{n}=28$ & Mujeres & NR & $\begin{array}{l}\text { Elite y } \\
\text { regionales }\end{array}$ & $\begin{array}{l}\text { Entrevista estructurada desarrollada } \\
\text { especificamente para examinar las } \\
\text { actividades de práctica de los atletas de } \\
\text { élite. }\end{array}$ & $\begin{array}{l}\text { Los deportistas acumularon en promedio unos } 13 \text { años y } 4000 \\
\text { horas en práctica concentrada específica del deporte antes de } \\
\text { alcanzar el estándar internacional. }\end{array}$ \\
\hline $\begin{array}{l}\text { Macnamara, et al. } \\
2016\end{array}$ & Varios deporte & s Metanalisis & $\mathrm{n}=34$ & NR & NR & $\begin{array}{l}\text { Elite- } \\
\text { Profesionales }\end{array}$ & Se utilizó la metodologia PRISMA & $\begin{array}{l}\text { Dispersión en las horas dedicadas al entrenamiento específico, } \\
\text { las medias ponderadas de práctica estructurada fueron } 3.9 \mathrm{~h} \\
(\mathrm{DE}=2.9 \mathrm{~h}), \text { y el promedio de horas de varió de } 4 \mathrm{~h} \text { a } 12.8 \\
\text { horas. En promedio, la práctica especializada representa el } 18 \% \\
\text { de la variación en el rendimiento deportivo, y el } 82 \% \\
\text { corresponde a otros factores. }\end{array}$ \\
\hline
\end{tabular}

to induce necesariamente a la práctica deportiva especializada, (niños en edades entre 6 y 12 años) la cual implica un proceso de entrenamiento continuo e intenso durante varios años, con un alto volumen de práctica en un solo depor- te y centrado en el rendimiento.Varios estudios apoyan este concepto en algunos deportes como, por ejemplo: gimnasia, (Law, Côté, \& Ericsson, 2008), fútbol (Ward, Hodges, Williams, \& Starkes, 2007; Ford, Ward, Hodges, \& Williams, 2009) ba-

Tabla 3

Especialización tempran

\begin{tabular}{|c|c|c|c|c|c|c|c|c|}
\hline Estudio & Deporte & $\begin{array}{l}\text { Tipo de } \\
\text { estudio }\end{array}$ & Población & Sexo & Edad & $\begin{array}{l}\text { Nivel } \\
\text { competitivo }\end{array}$ & Instrumentos & Principales resultados \\
\hline $\begin{array}{l}\text { Guellich \& Emrich, } \\
2006\end{array}$ & $\begin{array}{l}\text { Deportes } \\
\text { olímpicos }\end{array}$ & $\begin{array}{l}\text { Análisis } \\
\text { retrospectivo }\end{array}$ & $\mathrm{n}=1558$ & $\begin{array}{l}\text { Hombres y } \\
\text { mujeres }\end{array}$ & NR & Elite & $\begin{array}{l}\text { Cuestionario estandarizado, enviado a los } \\
\text { deportistas de veinte centros de } \\
\text { entrenamiento olímpico y devuelto vía } \\
\text { correo. }\end{array}$ & $\begin{array}{l}\text { Solo } 15 \text { de } 4972(0.3 \%) \text { de los seleccionados en edades muy } \\
\text { s jóvenes, se clasificó entre los } 10 \text { mejores atletas sénior } \\
\text { internacionales de alto nivel. Cuando la observación se realiza } \\
\text { en el tercer año, } 192 \text { atletas de } 11287 \text { de las escuelas de élite } \\
\text { deportivas (1.7\%) obtuvieron una medalla en un campeonato } \\
\text { internacional. }\end{array}$ \\
\hline $\begin{array}{l}\text { Guellich \& Emrich, } \\
2012\end{array}$ & $\begin{array}{l}\text { Diferentes } \\
\text { deportes }\end{array}$ & $\begin{array}{l}\text { Análisis } \\
\text { retrospectivo } \\
1999-2002\end{array}$ & $\mathrm{n}=4930$ & $\begin{array}{l}\text { Hombres y } \\
\text { mujeres }\end{array}$ & 20.6 & Elite & $\begin{array}{l}\text { En un grupo se utilizó la información de } \\
\text { los archivos de las federaciones } \\
\text { deportivas Alemanas sobre las carreras de } \\
\text { los deportistas y un seguimiento durante } \\
\text { tres años a los atletas a través de una } \\
\text { encuesta }\end{array}$ & $\begin{array}{l}\text { Observaron que cuanto más joven era reclutado el atleta al } \\
\text { e programa }(\mathrm{r}=0.92) \text {, más rápido era su abandono y por el } \\
\text { contrario cuando iniciaban a edades más tardías mayor era el } \\
\text { nivel de éxito logrado. }\end{array}$ \\
\hline Gullich, 2017 & $\begin{array}{l}\text { Diferentes } \\
\text { deportes }\end{array}$ & $\begin{array}{l}\text { Análisis } \\
\text { retrospectivo }\end{array}$ & $\mathrm{n}=166$ & $\begin{array}{l}\text { Hombres y } \\
\text { mujeres }\end{array}$ & 25 & $\begin{array}{l}\text { Elite junio y } \\
\text { sénior }\end{array}$ & $\begin{array}{l}\text { Cuestionario estandarizado, enviado a los } \\
\text { deportistas y devuelto vía correo. }\end{array}$ & $\begin{array}{l}\text { Los análisis revelaron que los medallistas se especializaron en } \\
\text { su deporte principal significativamente más tarde que los no } \\
\text { medallistas y acumularon significativamente menos práctica / } \\
\text { entrenamiento en el deporte principal durante la infancia / } \\
\text { adolescencia. }\end{array}$ \\
\hline $\begin{array}{l}\text { Gullich \& Emrich, } \\
2014\end{array}$ & $\begin{array}{l}\text { Diferentes } \\
\text { deportes }\end{array}$ & $\begin{array}{l}\text { Análisis } \\
\text { retrospectivo } \\
1999-2002\end{array}$ & $\mathrm{n}=1558$ & $\begin{array}{l}\text { Hombres y } \\
\text { mujeres }\end{array}$ & $\begin{array}{l}17.1- \\
24,4\end{array}$ & $\begin{array}{l}\text { Elite junior y } \\
\text { sénior }\end{array}$ & $\begin{array}{l}\text { Cuestionario estandarizado, enviado a los } \\
\text { deportistas y devuelto vía correo. }\end{array}$ & $\begin{array}{l}\text { El éxito juvenil no contribuyó a las diferencias individuales en } \\
\text { el éxito alcanzado a una edad avanzada } r=-0.09 \text {. Los atletas de } \\
\text { c clase mundial sénior se diferenciaron de los atletas de clase } \\
\text { nacional en una edad posterior para el inicio del entrenamiento } \\
\text { y la competencia en su dominio deportivo, especialización } \\
\text { posterior (14.4 vs. } 12.1 \text { años), }\end{array}$ \\
\hline Moesch, et al. 2011 & $\begin{array}{l}\text { Diferentes } \\
\text { deportes }\end{array}$ & $\begin{array}{l}\text { Análisis } \\
\text { retrospectivo }\end{array}$ & $n=243$ & $\begin{array}{l}\text { Hombres y } \\
\text { mujeres }\end{array}$ & 24.5 & Elite & $\begin{array}{l}\text { Cuestionario aplicado a través de un } \\
\text { enlace web, para verificar la validez de } \\
\text { los datos, aleatoriamente se realizó una } \\
\text { entrevista cuatro meses después. }\end{array}$ & $\begin{array}{l}\text { Los atletas de élite iniciaron su carrera más tarde que los } \\
\text { cercanos a la elite, también pasan por las diversas transiciones a } \\
\text { edades más tardías y tienen su primera competencia nacional o } \\
\text { internacional a una edad más avanzada. }\end{array}$ \\
\hline $\begin{array}{l}\text { Bridge \& Toms, } \\
2012\end{array}$ & $\begin{array}{l}\text { Diferentes } \\
\text { deportes }\end{array}$ & $\begin{array}{l}\text { Análisis } \\
\text { retrospectivo }\end{array}$ & $\mathrm{n}=2006$ & $\begin{array}{l}\text { Hombres y } \\
\text { mujeres }\end{array}$ & 25 & $\begin{array}{l}1006 \\
\text { deportistas del } \\
\text { Reino Unido }\end{array}$ & $\begin{array}{l}\text { Cuestionario estandarizado administrado } \\
\text { por email. }\end{array}$ & $\begin{array}{l}\text { La mayoría de los atletas que alcanzaron un nivel nacional de } \\
\text { competencia entre } 16-18 \text { años, no se especializaron en su } \\
\text { deporte principal desde edades tempranas, } 25 \% \text { lo hizo desde } \\
\text { los siete años, } 43 \% \text { desde los nueve, } 65 \% \text { a los once y } 86,7 \% \text { a } \\
\text { los trece años. }\end{array}$ \\
\hline Hall, et al. 2015 & $\begin{array}{l}\text { Baloncesto, } \\
\text { futbol y } \\
\text { voleibol }\end{array}$ & $\begin{array}{l}\text { Análisis } \\
\text { retrospectivo }\end{array}$ & $\mathrm{n}=546$ & Mujeres & NR & Elite & $\begin{array}{l}\text { Cuestionario estandarizado administrado } \\
\text { por email. }\end{array}$ & $\begin{array}{l}\text { El estudio reporto un aumento significativo } 1,5 \text { veces en el } \\
\text { dolor patelofemoral }(\mathrm{P}<0.038) \text { en los atletas que se } \\
\text { especializaron en un solo deporte y un riesgo relativo cuatro } \\
\text { veces mayor para las que practicaban un solo deporte en } \\
\text { comparación con atletas de deportes múltiples. }\end{array}$ \\
\hline Timothy et al., 2017 & $\begin{array}{l}\text { Diferentes } \\
\text { deportes }\end{array}$ & $\begin{array}{l}\text { Análisis } \\
\text { retrospectivo }\end{array}$ & $\mathrm{n}=1544$ & $\begin{array}{l}\text { Hombres y } \\
\text { mujeres }\end{array}$ & 16.1 & Elite junior & $\begin{array}{l}\text { Cuestionario estandarizado administrado } \\
\text { por email. }\end{array}$ & $\begin{array}{l}\text { La incidencia de las diversas lesiones fue mayor en los jóvenes } \\
\text { con niveles altos y moderados de especialización }(\mathrm{P}<0.03) \text { en } \\
\text { comparación a los que tenían bajos niveles de especialización. }\end{array}$ \\
\hline Post et al.2017 & $\begin{array}{l}\text { Diferentes } \\
\text { deportes }\end{array}$ & Caso control & $\mathrm{n}=2011$ & $\begin{array}{l}\text { Hombres y } \\
\text { mujeres }\end{array}$ & $12-18$ & $\begin{array}{l}\text { Regional- } \\
\text { Nacional }\end{array}$ & $\begin{array}{l}\text { Se utilizó un cuestionario que indago } \\
\text { sobre, el nivel de especialización, } \\
\text { volumen de participación deportiva anual } \\
\text { y semanal, e historial de lesiones. }\end{array}$ & $\begin{array}{l}\text { Identifico una asociación positiva entre la especialización } \\
\text { temprana y la historia de lesiones. Los atletas altamente } \\
\text { especializados reportaron mayor cantidad de lesiones de } \\
1 \text { cualquier tipo }(\mathrm{P}<0.001) \text { o una lesión por sobre uso }(\mathrm{P}<0.011) \\
\text { en comparación con los atletas del grupo de baja } \\
\text { especialización. }\end{array}$ \\
\hline Bush. Et al. 2019 & $\begin{array}{l}\text { Levantadores } \\
\text { de pesas }\end{array}$ & $\begin{array}{l}\text { Análisis } \\
\text { retrospectivo }\end{array}$ & $n=300$ & $\begin{array}{l}\text { Hombres y } \\
\text { mujeres }\end{array}$ & & Elite & $\begin{array}{l}\text { Cuestionario estandarizado administrado } \\
\text { por email (SurveyMonkey, San Mateo, } \\
\text { CA, USA) que indagaba sobre, edad de } \\
\text { especialización, lesiones y cirugías y } \\
\text { motivación para la especialización }\end{array}$ & $\begin{array}{l}\text { Hubo una diferencia estadísticamente significativa en la } \\
\text { ocurrencia de lesiones antes de los } 21 \text { años entre los } \\
\text { levantadores de pesas especializados tempranamente (p> } \\
0.0001 \text { ) La mayoría de los atletas ( } 68.8 \% \text { ) sintió que la } \\
\text { especialización temprana no era necesaria para alcanzar el } \\
\text { estatus de élite. Las lesiones ocurrieron con mayor frecuencia } \\
\text { en levantadores de pesas especializados en edades más jóvenes }\end{array}$ \\
\hline $\begin{array}{l}\text { McDonald, et al. } \\
2019\end{array}$ & $\begin{array}{l}\text { Lucha } \\
\text { Olimpica }\end{array}$ & $\begin{array}{l}\text { Análisis } \\
\text { retrospectivo }\end{array}$ & $n=143$ & NR & NR & Elite & $\begin{array}{l}\text { Cuestionario aplicado a través de un } \\
\text { enlace web (SurveyMonkey). }\end{array}$ & $\begin{array}{l}\text { El grupo de especialización temprana sufrió lesiones } \\
\text { significativamente más graves que el grupo de especialización } \\
\text { tardia }(\mathrm{P}<0.035) \text {. }\end{array}$ \\
\hline Knapik, et al. 2020 & Futbol & $\begin{array}{l}\text { Análisis } \\
\text { retrospectivo }\end{array}$ & $\mathrm{n}=64$ & Hombres & NR & Profesional & $\begin{array}{l}\text { Cuestionario aplicado a través de un } \\
\text { enlace web. }\end{array}$ & $\begin{array}{l}81 \% \text { de los atletas participaron en múltiples deportes antes de } \\
\text { especializarse y } 63 \% \text { se especializaron exclusivamente en el } \\
\text { fútbol. }\end{array}$ \\
\hline Buckley, et al. 2017 & $\begin{array}{l}\text { Beisbol, } \\
\text { Hockey }\end{array}$ & $\begin{array}{l}\text { Análisis } \\
\text { retrospectivo }\end{array}$ & $\mathrm{n}=3090$ & $\begin{array}{l}\text { Hombres y } \\
\text { mujeres }\end{array}$ & 23.6 & Elite & $\begin{array}{l}\text { Cuestionario aplicado a través de un } \\
\text { enlace web. }\end{array}$ & $\begin{array}{l}46 \% \text { de los atletas profesionales, }(\mathrm{P}<0.001) \text { se especializaron } \\
\text { en un solo deporte. La edad promedio de especialización fue a } \\
\text { los } 14.7 \text { años. Solo el } 22.3 \% \text { de los atletas profesionales dijeron } \\
\text { que querrían que su propio hijo se especializara en un solo un } \\
\text { deporte durante la infancia / adolescencia }\end{array}$ \\
\hline $\begin{array}{l}\text { Moseid, Myklebust } \\
2019\end{array}$ & Atletismo & $\begin{array}{l}\text { Análisis } \\
\text { retrospectivo } \\
2014-2015\end{array}$ & $\mathrm{n}=250$ & $\begin{array}{l}\text { Hombres y } \\
\text { mujeres }\end{array}$ & & Elite Junior & $\begin{array}{l}\text { Cuestionario aplicado a través de un } \\
\text { enlace web y entrevista }\end{array}$ & $\begin{array}{l}\text { Las diferencias en los puntajes de severidad acumulativa para } \\
\text { lesiones por uso excesivo, lesiones agudas y enfermedades } \\
\text { asociadas con la especialización temprana de un solo deporte. } \\
\text { Los análisis de regresión lineal mostraron que el puntaje de } \\
\text { severidad acumulativa no difirió significativamente entre las } \\
\text { categorías (datos no mostrados). }\end{array}$ \\
\hline
\end{tabular}


loncesto, (Leite \& Sampaio, 2012) debido a los altos niveles de experticia que se requieren, lo cual implicaría que estos atletas deban realizar de tres a siete veces más entrenamiento específico en comparación con atletas de otros deportes olímpicos. (Law et al., 2008). Sin embargo, la evidencia que soporta este planteamiento no es clara, es contradictoria e insuficiente y esta soportada principalmente en la experiencia y resultados en deportes como la gimnasia.

Los hallazgos de la presente revisión indican que menos del $1 \%$ de los deportistas que se especializaron en un deporte a temprana edad alcanzaron el nivel elite o profesional y los costos y riesgos potenciales asociados con la ET, conduce a menos disfrute de las actividades deportivas , restricción en la realización de otro tipo de actividades fuera del deporte, agotamiento, lesiones por uso excesivo, mayor riesgo de abandono y disminución en las carreras de los atletas Vaeyens et al (2009) (Tabla 3)

\section{Diversificación en el deporte}

Especializarse en un deporte competitivo a una edad temprana, o practicar una gama más amplia de deportes durante la adolescencia es un tema de debate, la diversificación en la práctica deportiva plantea que lo jóvenes atletas deben explorar una variedad de deportes. Los resultados de esta revisión indican que los atletas elite y súper elite de diversos deportes y países, se desempeñaron con un buen nivel de rendimiento en uno o varios deportes diferentes a su deporte principal. (Ver tabla 4) Estos hallazgos se confirman y son consistentes incluso cuando se comparan medallistas olímpicos, o de campeonatos mundiales, con no medallistas (Gullich, 2017; Rees et al., 2016), en diferentes países y tipos de deportes, y se reafirman en un estudio prospectivo de 3 años (Gullich \& Emrich, 2014)

Los estudios retrospectivos reportan que los atletas elite y súper elite, se desempeñaron con un buen nivel de rendimiento en un deporte diferente al principal (Vaeyens, Lenoir, Williams, \& Philippaerts, 2008) observando que un alto porcentaje de atletas de clase mundial entreno (60.9\%) y compitió $(47.2 \%)$ en otros deportes diferentes a su deporte principal actual, por lo tanto, invirtieron gran cantidad de tiempo de entrenamiento en otros deportes. Igualmente estos estudios indican que los atletas de clase mundial presentan mayor tendencia a practicar diversos deportes en comparación con sus homólogos de clase nacional (Gullich, 2014, 2017; Güllich \& Emrich, 2013; Hornig et al., 2016; Johnson, Tenenbaum, \& Edmonds, 2006; Moesch et al., 2011; Moesch, Hauge, Wikman, \& Elbe, 2013; Rees et al., 2016; Van Rossum, 2000)

La presente revisión indican que un alto porcentaje de los atletas de élite de diversos deportes como hockey sobre hielo, hockey de campo, baloncesto, béisbol, tenis, triatlón, remo y futbol, practicaron múltiples deportes a lo largo de su carrera deportiva. (Tabla 4)

\section{Discusión}

El objetivo de este artículo era revisar la literatura disponible sobre varios factores que han sido abordados desde múltiples estudios y considerados como fundamentales para lograr el éxito y llegar al deporte de elite. Los resultados indican un creciente interés de la comunidad científica a lo largo de los años para estudiar desde diversas perspectivas

\begin{tabular}{|c|c|c|c|c|c|c|c|c|}
\hline Estudio & Deporte & $\begin{array}{l}\text { Tipo de } \\
\text { estudio }\end{array}$ & Población & Sexo & Edad & $\begin{array}{l}\text { Nivel } \\
\text { competitivo }\end{array}$ & Instrumentos & Principales resultados \\
\hline Baker, et al.2003 & $\begin{array}{l}\text { Hockey } \\
\text { Baloncesto y } \\
\text { Netball }\end{array}$ & $\begin{array}{l}\text { Análisis } \\
\text { retrospectivo } \\
\text { Correlacional }\end{array}$ & $\mathrm{n}=28$ & Mujeres & NR & $\begin{array}{l}\text { Elite y } \\
\text { regionales }\end{array}$ & $\begin{array}{l}\text { Entrevista estructurada desarrollada } \\
\text { específicamente para examinar las } \\
\text { actividades de práctica de los atletas de } \\
\text { élite. }\end{array}$ & $\begin{array}{l}\text { Los atletas participaron en la práctica de otros deportes }(\mathrm{M}= \\
8.6, \mathrm{SD}=3.6) \text {. Los atletas que participaron en mayor cantidad } \\
\text { de diversas prácticas deportivas requirieron menos horas de } \\
\text { práctica deportiva especializada. }\end{array}$ \\
\hline Baker, et al. 2005 & $\begin{array}{l}\text { Triatletas } \\
\text { Iroman }\end{array}$ & Retrospectivo & $\mathrm{n}=28$ & $\begin{array}{l}\text { Hombres y } \\
\text { mujeres }\end{array}$ & 31.3 & Elite & $\begin{array}{l}\text { Entrevista estructurada desarrollada } \\
\text { específicamente para examinar las } \\
\text { actividades de práctica de los atletas }\end{array}$ & $\begin{array}{l}\text { Los triatletas más expertos tenían más horas }(8787.1 \mathrm{~h}) \text { de } \\
\text { entrenamiento en otros deportes, en comparación con los } \\
\text { 'middle of the pack } 5606.3 \mathrm{~h} \text { y los back of the pack } 4313.6 \mathrm{~h}\end{array}$ \\
\hline Berry, et al, 2009 & Futbol & $\begin{array}{l}\text { Análisis } \\
\text { retrospectivo }\end{array}$ & $\mathrm{n}=32$ & $\begin{array}{l}\text { Hombres y } \\
\text { mujeres }\end{array}$ & 25.2 & Elite & $\begin{array}{l}\text { Entrevista estructurada en profundidad } \\
\text { basada en la metodología de memoria } \\
\text { retrospectiva para examinar las historias } \\
\text { deportivas y prácticas relacionadas de los } \\
\text { atletas }\end{array}$ & $\begin{array}{l}\text { Los jugadores de mayor rendimiento diferían de los menos } \\
\text { hábiles en haber participado significativamente en más en } \\
\text { deportes (3.6) de invasión, que los de menor rendimiento (2.5) } \\
\text { acumulado, más horas de experiencia en este tipo de } \\
\text { actividades. }\end{array}$ \\
\hline $\begin{array}{l}\text { Bridge \& Toms, } \\
2012\end{array}$ & $\begin{array}{l}\text { Diferentes } \\
\text { deportes }\end{array}$ & $\begin{array}{l}\text { Análisis } \\
\text { retrospectivo }\end{array}$ & $\mathrm{n}=2006$ & $\begin{array}{l}\text { Hombres y } \\
\text { mujeres }\end{array}$ & 25 & Elite & $\begin{array}{l}\text { Cuestionario estandarizado, administrado } \\
\text { por email }\end{array}$ & $\begin{array}{l}\text { Encontró una asociación significativa entre el número de } \\
\text { deportes practicados y un nivel más elevado de rendimiento } \\
\text { Los deportistas que compitieron en tres deportes fueron } \\
\text { significativamente más propensos a competir a nivel nacional } \\
\text { que aquellos que practicaron un solo deporte }\end{array}$ \\
\hline Gullich, 2014 & Hockey & $\begin{array}{l}\text { Análisis } \\
\text { retrospectivo }\end{array}$ & $\mathrm{n}=54$ & Hombres & 26.6 & Elite & $\begin{array}{l}\text { Cuestionario estandarizado, enviado a los } \\
\text { deportistas y devuelto vía correo. }\end{array}$ & $\begin{array}{l}\text { Los análisis revelaron que los Campeones Olímpicos realizaron } \\
\text { volúmenes moderados de entrenamiento y obtuvieron su } \\
\text { primera medalla internacional de alto nivel después de } \\
\text { acumular } 4393 \pm 1389 \text { y se dedicaron a otras actividades } \\
\text { deportivas extensas durante la infancia y la juventud. }\end{array}$ \\
\hline $\begin{array}{l}\text { Gullich \& Emrich, } \\
2014\end{array}$ & $\begin{array}{l}\text { Diferentes } \\
\text { deportes }\end{array}$ & $\begin{array}{l}\text { Análisis } \\
\text { retrospectivo }\end{array}$ & $\mathrm{n}=1558$ & $\begin{array}{l}\text { Hombres y } \\
\text { mujeres }\end{array}$ & $\mathrm{NR}$ & Elite & $\begin{array}{l}\text { Cuestionario estandarizado, enviado a los } \\
\text { deportistas y devuelto vía correo. }\end{array}$ & $\begin{array}{l}\text { Los deportistas de clase mundial diferían de la clase nacional } \\
\text { en mayor participación en otros deportes (entrenamiento } 66 \% \\
\text { vs. } 51 \% \text { y competiciones } 53 \% \text { vs. } 39 \% \text { ) Los resultados se } \\
\text { confirmaron con pruebas longitudinales y fueron ampliamente } \\
\text { consistentes en todos los tipos de deportes. }\end{array}$ \\
\hline $\begin{array}{l}\text { Guellich \& } \\
\text { Emrich, } 2006\end{array}$ & $\begin{array}{l}\text { Deportes } \\
\text { olimpicos }\end{array}$ & $\begin{array}{l}\text { Análisis } \\
\text { retrospectivo }\end{array}$ & $\mathrm{n}=1558$ & $\begin{array}{l}\text { Hombres y } \\
\text { mujeres }\end{array}$ & 22.5 & Elite & $\begin{array}{l}\text { Cuestionario estandarizado, enviado a los } \\
\text { deportistas y devuelto vía correo. }\end{array}$ & $\begin{array}{l}\text { Los atletas internacionalmente exitosos mantienen el } \\
\text { entrenamiento en otros deportes durante mucho más tiempo en } \\
\text { comparación con los atletas menos exitosos }(13.3 \pm 5.6 \text { vs. } 11.8 \\
\pm 5.1 \text { años } \mathrm{p}<0.05) \text {. El } 64 \% \text { de los finalistas internacionales y } \\
\text { el } 53 \% \text { de los atletas de alto nivel menos exitosos, han } \\
\text { participado en otros deportes. }(\mathrm{p}<0.01)\end{array}$ \\
\hline $\begin{array}{l}\text { Fraser-Thomas et } \\
\text { al., } 2008\end{array}$ & Natación & $\begin{array}{l}\text { Análisis } \\
\text { retrospectivo }\end{array}$ & $\mathrm{n}=50$ & $\begin{array}{l}\text { Hombres y } \\
\text { mujeres }\end{array}$ & 18.7 & Elite & $\begin{array}{l}\text { Cuestionario estandarizado, enviado a los } \\
\text { deportistas y devuelto vía correo. }\end{array}$ & $\begin{array}{l}\text { Los nadadores más exitosos presentaron significativamente } \\
(\mathrm{p}<0.05) \text { una mayor participación en la práctica de otros } \\
\text { deportes que los menos exitosos, y los nadadores que } \\
\text { abandonaron el entrenamiento participaron en } \\
\text { significativamente menos actividades deportivas que los atletas } \\
\text { más exitosos. }\end{array}$ \\
\hline Guillich 2018 & Atletismo & $\begin{array}{l}\text { Análisis } \\
\text { retrospectivo }\end{array}$ & $\mathrm{n}=264$ & $\begin{array}{l}\text { Hombres y } \\
\text { mujeres }\end{array}$ & 21.6 & Elite & $\begin{array}{l}\text { Cuestionario estandarizado, administrado } \\
\text { por email }\end{array}$ & $\begin{array}{l}\text { Los atletas de mayor nivel tuvieron mayor participación en } \\
\text { prácticas organizadas y competencias en otros deportes en } \\
\text { comparación con atletas de nivel más bajo. Igualmente, } \\
\text { acumularon más horas de práctica en otros deportes antes de } \\
\text { comenzar el atletismo y también continuaron practicando y } \\
\text { compitiendo en otros deportes durante más años que los atletas } \\
\text { de nivel más bajo y se especializaron en atletismo a una edad } \\
\text { posterior, principalmente en la adolescencia tardía }\end{array}$ \\
\hline
\end{tabular}


estos factores. En las siguientes secciones se discuten algunos de los resultados más interesantes que surgen de los análisis realizados en esta revisión, desde una perspectiva teórica.

\section{Efectos de la edad relativa}

La influencia de la ERE en la detención de talentos es un fenómeno que se presenta en todos los deportes como se observó en los resultados de esta revisión. Se identificaron varios aspectos sobre los cuales puede influir el ERE y que han sido suficientemente documentados: a) En los procesos de selección los jóvenes nacidos al inicio del año o los maduradores precoces, tienen mayores posibilidades de ser seleccionados por los entrenadores para niveles más altos de competencia en comparación con los que nacen a finales del mismo año (Gutierrez Diaz Del Campo, Pastor Vicedo, Gonzalez Villora, \& Contreras Jordan, 2010; Müller, Hildebrandt, \& Raschner, 2015; Nakata, 2017b; Romann \& Cobley, 2015), y son considerados erróneamente como más talentosos, cuando en realidad su mejor rendimiento y desempeño se debe a que son físicamente y cognitivamente más maduros que sus homólogos de edad cronológica más jóvenes. ( Helsen et al., 2005) b) cuando se evalúan sus condiciones físicas y deportivas se presenta una diferencia en su rendimiento que podría ser causada, por la existencia de grandes diferencias inter-individuales en los procesos de desarrollo y maduración (físico, neurológico psicologico) y de experiencia de vida. (Cobley et al., 2009)

Las dimensiones corporales, las cualidades físicas, las capacidades condicionales, y las habilidades psicológicas técnicas y tácticas están vinculadas al desarrollo y la maduración y proporcionan ventajas en el rendimiento de la mayoría deportes. El estado de maduración neurológica puede manifestarse en diferentes capacidades como la autorregulación de la atención, la emoción y otras funciones como memoria selectiva, atención, ciertos aspectos de la metacognición y control de la inhibición.(Gutierrez Diaz Del Campo et al., 2010) En relación con las diferencias psicológicas también se han sugerido disparidades. (Musch \& Grondin, 2001) Los deportistas relativamente mayores pueden tener mayores probabilidades de desarrollar percepciones más elevadas de competencia (Harter, 1978) y autoeficacia, en comparación con los atletas relativamente más jóvenes que pueden ser más propensos a tener experiencias deportivas negativas, desarrollar percepciones de baja competencia que lo pueden conducir a abandonar la práctica deportiva.(Smith, 1986)

En resumen si bien es cierto existen divergencias entre los diferentes estudios que se han realizado sobre el ERE, se observa una tendencia clara con evidencias empíricas que demuestra la influencia que tiene el ERE en los procesos de selección y desarrollo de talentos, lo que pondría en tela de juicio los sistemas actuales de organización y competición por edades cronológicas, tanto en la participación deportiva de base, como en los sistemas de detección y desarrollo de atletas. Para ello se han realizado varias propuestas : (1) diseñar calendarios con límites de edad alternativos de selección (Helsen, Winckel, \& Williams, 2005); (2) crear más categorías de edad con rangos más pequeños (Lopez de Subijana \& Lorenzo, 2018); (3) dividir a los jugadores en categorías se- gún el nivel de habilidad (Sarmento, Anguera, Pereira, \& Araujo, 2018) ; y (4) permitir que los jugadores nacidos en los últimos meses del año cambien temporalmente a una categoría de edad más joven(González-Víllora, Pastor-Vicedo, \& Cordente, 2015).

\section{Volumen de entrenamiento}

La relación VE y éxito deportivo de elite fue argumentada desde los siguientes supuestos: (a) el éxito internacional en el deporte de élite es el resultado de carreras a largo plazo «lineales» en una disciplina deportiva; (b) el éxito aumenta con la duración prolongada del entrenamiento y de la práctica en este deporte; y (c) inicio temprano en el entrenamiento, éxito temprano, inicio rápido en la competición y continuación en los programas de promoción. (Guellich \& Emrich, 2006).

Aunque son pocos los estudios que abordan la importancia del VE, existe una clara evidencia que no existe correlación entre este y el éxito deportivo de elite.

Análisis retrospectivos de los perfiles históricos en atletas de elite de diferentes deportes (por ejemplo, hockey de campo, patinaje artístico, fútbol y lucha libre) revelaron que estos sujetos realizaron VE considerables.(Le Gall, Carling, Williams, \& Reilly, 2010). Independientemente de la amplia variación entre los deportes, la mayoría de los atletas élite, y súper élite acumulan enormes volúmenes de entrenamiento, convirtiendo la práctica extensiva del deporte, en un requisito previo para el rendimiento de clase mundial. (Weissensteiner, Abernethy, Farrow, \& Muller, 2008)

Sin embargo, a pesar de la existencia de estos estudios que apoyan una relación positiva entre la cantidad de entrenamiento y el rendimiento de élite, pocos han demostrado que 10.000 horas de práctica intencionada son un requisito previo para alcanzar el alto rendimiento (Helsen, Starkes, \& Hodges, 1998; Starkes, 2000). Esta evidencia se ratifica en el metanalisis realizado por Macnamara, B., Moreau, D., \& Hambrick, D. (2016) que indican, una gran dispersión en las horas dedicadas al entrenamiento específico, las medias ponderadas de práctica deliberada fueron 3,949 h $(\mathrm{DE}=2,942 \mathrm{~h})$, y el promedio de horas de varió de 4 h a 12,839 horas. Igualmente la revisión realizada por Rees et al., (2016) indica que la calidad de la evidencia que soporta la aplicabilidad de la regla de 10 años / $10.000 \mathrm{~h}$, está limitada por la calidad moderada de los estudios. Sin embargo estos resultados son cuestionados por Ericson A (2020) quien realiza un metanalisis e indica que los resultados del estudio Macnamara, B., Moreau, D., \& Hambrick, D. (2016) debido a la interpretación equivocada de «práctica deliberada» y estimó que la duración acumulada de la práctica explicaba una variación considerablemente mayor en el rendimiento ( 29 y $61 \%$ )

Sin embargo a pesar lo observado por Ericson los resultados de la presente revisión donde se tuvo bien definido el concepto de práctica estructurada, indican que existe una fuerte evidencia que demostró que en la mayoría de los deportes el éxito de los deportistas de elite y súper elite, no se encuentra correlacionado con altos volúmenes de entrenamiento.

\section{Especialización temprana}

La ET se basa en el supuesto que concentrarse en un 
deporte desde temprana edad es un factor determinante para obtener mejores resultados y lograr el alto rendimiento en la edad adulta. Plantea que el rendimiento máximo en el deporte se logra antes de la madurez biológica, razón por la cual una especialización temprana es necesaria y único camino para alcanzar la élite deportiva. Sin embargo, a pesar de no haberse podido demostrar de forma concluyente esta relación, las tasas de especialización deportiva a nivel mundial son elevadas y continúan con un alto incremento, por ejemplo en Estados Unidos la participación de niños de d» 6 años paso del 6\% en 1997 al 12\% en 2008 y el 70\% de los niños abandonan la práctica deportiva organizada a los 13 años. (Jayanthi et al., 2013) La Asociación de Tenis de EE. UU, encontró que el $70 \%$ de los participantes comenzó a especializarse a una edad promedio de 10.4 años. (Jayanthi, Dechert, Durazo, Dugas, \& Luke, 2011a)

La evidencia científica revisada en este artículo sugiere que retrasar la especialización deportiva para la mayoría de los deportes hasta después de la pubertad ( $<\ll 15$ o 16) minimizaría los riesgos y conducirá a una mayor probabilidad de éxito atlético a nivel sénior.( Malina, 2010; Mostafavifar et al., 2013 ;Jayanthi et al., 2015 ;Brenner, 2016). Estos resultados se ratifican en el metaanlisis relizado por Macnamara, B., Moreau, D., \& Hambrick, D. (2016) que indican que en promedio, la práctica especializada representa el 18\% (estos valores varias según el deporte) de la variación en el rendimiento deportivo, y el $82 \%$ de la variación corresponde a otras factores.

La realidad del deporte de elite (con excepción de algunos deportes como la gimnasia y el patinaje artístico) aporta evidencia contundente que indica que las probabilidades de sobresalir en la elite deportiva, no parecen aumentar con la ET (LaPrade et al., 2016), por el contrario sugiere que la especialización tardía en un deporte en particular, puede generar mejores resultados que la especialización temprana.

Martindale, Collins, \& Daubney, (2005) plantea que si el $90 \%$ de los mejores 25 atletas del mundo no fueron exitosos a edades tempranas, que necesidad existe de involucrar al niño en el alto rendimiento a edades tempranas? Si bien es cierto que la mayoría de atletas de élite y súper-elite participaron en programas de desarrollo en algún momento de su carrera deportiva, hay evidencia que el éxito en las categorías infantil y juvenil no predice significativamente el éxito en las categorías mayores.(Guellich \& Emrich, 2006; Moesch et al., 2013; Vaeyens et al., 2009; Gullich \& Emrich, 2014) Por ejemplo, en el fútbol de Inglaterra, solo dos jugadores han participado internacionalmente en las diferentes categorías de edad, incluido el nivel sénior, Michael Owen y Terry Venables. Esto se ratifica con varios estudios que demostraron que el éxito competitivo a nivel juvenil e infantil no se correlaciona (o modestamente en el mejor de los casos) con el éxito a nivel sénior. (Barreiros, Cote, \& Fonseca, 2014; Brouwers, Bosscher, \& Sotiriadou, 2012; Gullich \& Emrich, 2014; Vaeyens et al., 2009)

Esta serie de evidencias ponen en cuestión la eficacia y el valor de la especialización deportiva precoz. (Baker, Cobley, \& Fraser-Thomas, 2009; Bean, Fortier, Post, \& Chima, 2014 ; Feeley et al., 2016 ; Malina, 2010 ;Pankhurst, Collins, \& Macnamara, 2013)

Los hallazgos de esta y de revisiones previas y declara- ciones de diversos organismos internacionales del deporte y la salud, indican los efectos potencialmente negativos de la especialización temprana y el impacto positivo de experiencias juveniles diversificadas entre deportes y entornos. Para la mayoría de los deportes, la especialización tardía con diversificación temprana presenta mayores probabilidades conducir a la élite deportiva. (Brenner, Small, \& Bernhardt, 2007; Brenner, 2016, Jayanthi, et al 2013; Côté, Horton, MacDonald, \& Wilkes, 2009; Côté et al., 2011; DiFiori et al., 2014; Faigenbaum et al., 2009; LaPrade et al., 2016; Myer et al., 2015; Rees et al., 2016) En resumen la evidencia indica que la especialización temprana no es necesaria para el desarrollo de los atletas de élite, por el contrario antes de la adolescencia, la práctica de diversos deportes es más importante (Cote \& Hay, 2002; Hill, 1993)y puede ser la base para el fortalecimiento de la mentalidad y habilidades físicas(Ericsson, 1998)

\section{Diversificación en la práctica deportiva}

Aunque pocos estudios han abordado la importancia de la DPD, existe una clara asociación entre esta y el deporte de elite. Los resultados de la presente revisión indican que la diversificación temprana, a pesar de la reducción que genera en el volumen de entrenamiento en el deporte específico, presenta efectos positivos. Gullich et al., (2017); Wright \& Côté, (2003) demostraron que las experiencias deportivas diversificadas durante la infancia, fomentan relaciones positivas con los compañeros y habilidades de liderazgo. Los estudios longitudinales indican que los jóvenes que participan en actividades deportivas variadas obtienen mejores resultados personales y sociales, como el bienestar (Busseri, Rose-Krasnor, Willoughby, \& Chalmers, 2006) las relaciones positivas con los compañeros ( Fredricks \& Eccles, 2006) y la motivación (Hendry, Crocker, \& Hodges, 2014), e igualmente están asociadas a una mayor posibilidad de éxito deportivo posterior. (Baker et al., 2003; Carlson, 1988; Guellich \& Emrich, 2006; Helsen et al., 1998; Law et al., 2008; Moesch et al., 2011; Soberlak \& Cote, 2003; Wall \& Côté, 2007) Varios fundamentos teóricos soportan estos planteamientos. Las habilidades perceptivo-motoras, psicológicas y las capacidades fisiológicas desarrolladas son transferibles entre deportes con características similares (Abernethy, Baker, \& Côté, 2005; Côté et al., 2007; Rea \& Lavallee, 2015). Las experiencias tempranas de aprendizaje en diversos deportes genera varios efectos positivos en los jóvenes atletas: a) mejoran la eficacia de la práctica en el deporte específico, obteniendo más rápidamente un buen nivel el rendimiento invirtiendo menor tiempo en la práctica del deporte especifico.(Carlson, 1988; Gullich, 2014, 2017; Gullich \& Emrich, 2014; Hornig et al., 2016); b) facilitan los procesos de adquisición y afinamiento de habilidades específicas.(Vaeyens et al., 2009) Los atletas adquieren un repertorio multifacético en términos de una «red» más amplia de habilidades motoras perceptivas, que favorecen respuestas funcionales que permite a los jóvenes resolver de forma eficiente situaciones motoras inmediatas. (Ford \& Williams, 2012; Wolpert, Diedrichsen, \& Flanagan, 2011); c) la experiencia en varios deportes aumenta la probabilidad de que un atleta elija el deporte en el que tiene más talento (Gullich \& Emrich, 2014) 


\section{Conclusiones}

Desde hace varias décadas, ha habido un creciente interés por indagar sobre algunos aspectos que han sido considerados fundamentales para el éxito en los deportistas de elite. La gran cantidad de estudios revisados aquí permitió la identificación de los temas abordados con mayor frecuencia en esta área de investigación: efectos relativos de la edad, volumen de entrenamiento, especialización temprana y diversificación en el entrenamiento.

La evidencia revisada indicó que los atletas de elite y súper elite en la mayoría de los deportes no necesariamente fueron detectados a edades tempranas, por lo tanto no se especializaron en un deporte específico de forma prematura y por el contrario practicaron alternativamente varios deportes. Todo esto condujo a que tuvieran un volumen de entrenamiento específico en el deporte por debajo de las 10000 horas.

La literatura revisada destacó que existe una relación compleja entre estos factores, que debe ser tenida en cuenta por las personas que están involucradas en el proceso de identificación y desarrollo de los deportistas talentosos para que establezcan un equilibrio óptimo entre la edad de selección del talento, el momento de la especialización, el volumen total de entrenamiento y la práctica deportiva diversificada, por la estrecha relación que estos factores tienen los niveles más altos de rendimiento.

Para comprender y tener suficientes y mejores argumentos científicos, se requiere mayor cantidad de estudios longitudinales que sigan la trayectoria completa de los deportistas más exitosos.

Otra brecha de investigación identificada en esta revisión, es la ausencia de análisis multidimensionales que determinen la interacción de estos factores y su influencia en el éxito en los deportistas de elite y súper elite.

\section{Referencias}

Abbott, A., \& Collins, D. (2002). A theoretical and empirical analysis of a 'state of the art' talent identification model. High Ability Studies, 13(2), 157-178. doi: 10.1080/ 1359813022000048798

Abernethy, B., Baker, J., \& Côté, J. (2005). Transfer of pattern recall skills may contribute to the development of sport expertise. Applied Cognitive Psychology, 19 (3) 705-718 doi: 10.1002/acp.1102

American Academy of Pediatrics (1991). Sport Medicine: Health Care for Young Athletes (2nd ed.). Elk Grove Village.

Arrieta H, Torres-Unda J, Gil SM, Irazusta J. Relative age effect and performance in the U16, U18 and U20 European Basketball Championships. Journal of sports sciences, 2016; 34(16): 1530-1534

Augste, C., \& Lames, M. (2011). The relative age effect and success in German elite U-17 soccer teams. Journal of sports sciences, 29(9), 983-987. doi: 10.1080/ 02640414. 2011.574719

Baker, J., Janning, C., Wong, H., Cobley, S., \& Schorer, J. (2014). Variations in relative age effects in individual sports: skiing, figure skating and gymnastics. European journal of sport science, 14 Suppl 1, S183-190. doi:
10.1080/17461391.2012.671369

Baker, J., Cobley, S., \& Fraser-Thomas, J. (2009). What do we know about early sport specialization? not much! High Ability Studies, 20(1), 77-89.

Baker, J., \& Logan, A. J. (2007). Developmental contexts and sporting success: birth date and birthplace effects in national hockey league draftees 2000-2005. British Journal of Sports Medicine, 41(8), 515-517. doi: 10.1136/ bjsm.2006.033977

Baker, J., Côté, J., \& Deakin, J. (2005). Expertise in ultraendurance triathletes early sport involvement, training structure, and the theory of deliberate practice. Journal of Applied Sport Psychology 17(1) 64-78. doi.org/10.1080/ 10413200590907577

Baker, J., Cote, J., \& Abernethy, B. (2003). Sport-specific practice and the development of expert decision-making in team ball sports. Journal of Applied Sport Psychology, 15(1), 12-25. doi: 10.1080/10413200305400

Barreiros, A., Cote, J., \& Fonseca, A. M. (2014). From early to adult sport success: analysing athletes' progression in national squads. European journal of sport science, 14 Suppl 1, S178-182. doi: 10.1080/17461391.2012.671368

Bean, C., Fortier, M., Post, C., \& Chima, K. (2014). Understanding how organized youth sport may be harming individual players within the family unit: a literature review. International Journal of Environmental Research and Public Health, 11(10), 10226-10268. doi: 10.3390/ijerph111010226

Berry, J., Abernethy, B., \& Côté, J. (2009). The contribution of structured activity and deliberate play to the development of expert perceptual and decision-making skill. Journal of sport \& exercise psychology 30(6)685-708. DOI:10.1123/ jsep.30.6.685

Bompa, T. (1994). Theory and methodology of training: the key to athletic training. Champaign: Human Kinetics.

Brenner, J. S. (2016). Sports specialization and intensive training in young athletes. Pediatrics, 138(3). doi: 10.1542/peds.2016-2148

Brenner, J., Small, E., \& Bernhardt, D. (2007). American Academy of Pediatrics Council on Sports Medicine and Fitness. Pediatrics, 119(6), 1242-1245.

Bridge, M., \& Toms, M. (2012). The specialising or sampling debate: A retrospective analysis of adolescent sports participation in the UK. Journal of sports sciences 31(1) 87-96. DOI:10.1080/02640414.2012.721560

Brouwers, J., Bosscher, V., \& Sotiriadou, P. (2012). An examination of the importance of performances in youth and junior competition as an indicator of later success in tennis. Sport Management Review, 15, 461-475. doi: 10.1016/j.smr.2012.05.002

Brustio,P.,Kearney,P., Lupo,C., Ungureanu,A., Mulasso,A.,Rainoldi,A.,\&Boccia,G.(2019) Relative Age Influences Performance of World-Class Track and Field Athletes Even in the Adulthood. Frontiers in Psychology. 10,1395

Bullock, N., Gulbin, J., Martin, D., Ross, A., Holland, T., \& Marino, F. (2009). Talent identification and deliberate programming in skeleton: ice novice to Winter Olympian in 14 months. Journal of sports sciences 27(4) 397-404 DOI:10.1080/02640410802549751 
Bush, C., Wilhelm, A., Lavallee, M.,\& Deitch, J. (2019) Early Sport Specialization in Elite Weightlifters: Weightlifting Injury Occurrence and Relevant Opinion. [published online ahead of print, 2019 Jul 1]. J Strength Cond Res. 2019;10.1519/ JSC.0000 000000003239. doi:10.1519/ JSC.0000000000003239

Busseri, M., Rose-Krasnor, L., Willoughby, T., \& Chalmers, H. (2006). A longitudinal examination of breadth and intensity of youth activity involvement and successful development. Developmental psychology, 42(6), 13131326. doi: 10.1037/0012-1649.42.6.1313

Campos,F., Pellegrinotti, Í., Campos, L., Dias, T,. Gómez M.(2020) Relative Age Effect in the Girls'Volleyball U18 World Championship. Journal of Human Kinetics 72 (1), 195-202

Carlson, R. (1988). The socialization of elite tennis players in sweden: an analysis of the players' backgrounds and development. Sociology of Sport Journal 5(3), 241-256 doi: $10.1123 /$ ssj.5.3.241

Cobley, S., Baker, J., Wattie, N., \& McKenna, J. (2009). Annual age-grouping and athlete development: a meta-analytical review of relative age effects in sport. Sports medicine, 39(3),235-256. doi: 10.2165/00007256-200939030-00005

Cochrane Consumers and Communication Review Group. (2016). Data extraction template for included studies. .

Connor, J., Renshaw, I., \& Doma, K. (2019). Moderating factors influence the relative age effect in Australian cricket. PEREJ, 7, e6867. doi: 10.7717/pEREj.6867

Côté, J., Lidor, R., \& Hackfort, D. (2011). ISSP position stand: To sample or to specialize? Seven postulates about youth sport activities that lead to continued participation and elite performance. International Journal of Sport and Exercise Psychology 7(1) 7-16 DOI: 10.1080/ 1612197X.2009.9671889

Côté, J., Horton, S., MacDonald, D., \& Wilkes, S. (2009). The Benefits of Sampling Sports During Childhood. Physical \& Health Education Journal, 74, 6-11.

Côté, J., Lidor, R., \& Hackfort, D. (2009). ISSP position stand: To sample or to specialize? Seven postulates about youth sport activities that lead to continued participation and elite performance. International Journal of Sport and Exercise Psychology, 7(1), 7-17. doi: 10.1080/ 1612197X.2009.9671889

Cote, J., \& Hay, J. (2002). Children $1 / 4$ S involvement in sport: A developmental perspective. In J. M. S. D. Stevens (Ed.), Psychological foundations of sport (pp. 484-502). Boston: Merrill.

Cumming, S. P., Searle, C., Hemsley, J. K., Haswell, F., Edwards, H., Scott, S., . . Malina, R. M. (2018). Biological maturation, relative age and self-regulation in male professional academy soccer players: A test of the underdog hypothesis. Psychology of Sport and Exercise, 39, 147-153. doi: https://doi.org/10.1016/ j.psychsport.2018.08.007

Deakin, J. M., \& Cobley, S. (2003). A search for deliberate practice. An examination of the practice environments in figure skating and volleyball. In J. L. Starkes \& K. A. Ericsson (Eds.), Expert performance in sports. Advances in research on sport expertise (pp. 115-135). Champaign, IL: Human Kinetics
De Bosscher, V., De Knop, P., Van Bottenburg, M., \& Shibli, S. (2006). A Conceptual Framework for Analysing Sports Policy Factors Leading to International Sporting success. European Sport Management Quarterly, 6(2), 185-215. doi: 10.1080/16184740600955087

DiFiori, J., Benjamin, J., Brenner, J., Gregory, A., Jayanthi, N., Landry, G., \& Luke, A. (2014). Overuse injuries and burnout in youth sports: a position statement from the American Medical Society for Sports Medicine. British journal of sports medicine, 48(4), 287-288. doi: 10.1136/bjsports2013-093299

Edgar, S., \& O'Donoghue, P. (2005). Season of birth distribution of elite tennis players. Journal of sports sciences, 23(10), 1013-1020. doi: 10.1080/ 02640410400021468

Ericsson, K. A. (2020) Towards a science of the acquisition of expert performance in sports: Clarifying the differences between deliberate practice and other types of practice. Journal of sports sciences. 38(2) 159-176.

Ericsson, K., Krampe, R. T., \& Tesch-Romer, C. (1993). The role of deliberate practice in the acquisition of expert performance. Psychological Review,100(3) 363-406. Doi: 10.1037//0033-295X.100.3.363

Ericsson, K. (1998). The scientific study of expert levels of performance general. implications for optimal learning and creativity. High Ability Studies 9(1) 73-100) doi.org/ 10.1080/1359813980090106

Faber, I., Bustin, P., Oosterveld, F., Elferink-Gemser, M., \& Nijhuis-Van der Sanden, M. (2016). Assessing personal talent determinants in young racquet sport players: a systematic review. Journal of sports sciences, 34(5), 395410. doi:: 10.1080/02640414.2015.1061201

Faigenbaum, A., Kraemer, W., Blimkie, C., Jeffreys, I., Micheli, L., Nitka, M., \& Rowland, T. (2009). Youth resistance training: updated position statement paper from the national strength and conditioning association. Journal of strength and conditioning research, 23(5 Suppl), S6079. doi:: 10.1519/JSC.0b013e31819df407

Feeley, B.,Agel, J., \& LaPrade, R. (2016). When is it too early for single sport specialization? The American journal of sports medicine , 44(1), 234-241. doi: 10.1177/ 0363546515576899

Ferriz-Valero, A., Pérez, S., Cejuela, R.,\& García-Jaén, M. (2019) Efecto de la Edad Relativa para el Desarrollo del Talento en Jóvenes Triatletas. Retos, 37(37), 27-32.

Ford, P., \& Williams, A. (2012). The developmental activities engaged in by elite youth soccer players who progressed to professional status compared to those who did not. Psychology of Sport and Exercise - PSYCHOL SPORT EXERC, 13. doi: 10.1016/j.psychsport.2011.09.004

Ford, P., Ward, P., Hodges, N., \& Williams, A. (2009). The role of deliberate practice and play in carERE progression in sport: the early engagement hypothesis. High Ability Studies, 20(1), 65-75. doi:10.1080/13598130902860721

Fraser-Thomas, J., Côté, J., \& Deakin, J. (2008). Examining adolescent sport dropout and prolonged engagement from a developmental perspective. Journal of Applied Sport Psychology 20(3):318-333. doi: 10.1080/ 10413200802163549

Fredricks, J., \& Eccles, J. (2006). Extracurricular involvement 
and adolescent adjustment: impact of duration, number of activities, and breadth of participation. Applied developmental Science, 10 (3) 132-146.doi: 10.1207/ s1532480xads1003_3

Gerrard, D.E (1993) Overuse injury and growing bones: the young athlete at risk. British journal of sports medicine .27: 14-18,

González-Víllora, S., Pastor-Vicedo, J. C., \& Cordente, D. (2015). Relative Age Effect in UEFA Championship Soccer Players, Journal of Human Kinetics, 47(1), 237. doi:10.1515/hukin-2015-0079

Gullich, A., Kovar, P., Zart, S., \& Reimann, A. (2017). Sport activities differentiating match-play improvement in elite youth footballers - a 2-year longitudinal study. Journal of sports sciences, 35(3), 207-215. doi: 10.1080/ 02640414.2016 .1161206

Gullich, A. (2017). International medallists' and nonmedallists' developmental sport activities - a matchedpairs analysis. Journal of sports sciences, 35(23), 22812288. doi: 10.1080/02640414.2016.1265662

Gullich,A. (2014). Many roads lead to rome - developmental paths to olympic gold in men's field hockey. European journal of sport science, 14(8), 763-771. doi: 10.1080/ 17461391.2014.905983

Gullich, A., \& Emrich, E. (2014). Considering long-term sustainability in the development of world class success. European journal of sport science, 14 Suppl 1, S383397. doi: $10.1080 / 17461391.2012 .706320$

Güllich, A., \& Emrich, E. (2013). Investment patterns in the carEREs of elite athletes in east and west Germany. European Journal for Sport and Society, 10(3), 191-214. doi: 10.1080/16138171.2013.11687919

Guellich, A., \& Emrich, E. (2012). Individualistic and collectivistic approach in athlete support programmes in the german high-performance sport system european. Journal for Sport and Society 9, 4, S. 243-268

Guellich,A., \& Emrich, E. (2006). Evaluation of the support of young athletes in the elite sport system. European Journal for Sport and Society, 3 (2), 85-108. doi: 10.1080/ 16138171.2006.11687783

Gustin, W C. (1985). The development of exceptional research mathematicians.

In B. S. Bloom (Ed), Developing talent in young people (pp. 270-331). New York: Ballantine Books.

Gutierrez Diaz Del Campo, D., Pastor Vicedo, J., Gonzalez Villora, S., \& Contreras Jordan, O. (2010). the relative age effect in youth soccer players from spain. Journal of Sports Science \& Medicine, 9(2), 190-198. Doi 10.1371/ journal.pone.0192209

Hall, R., Foss, K. B., Hewett, T. E., \& Myer, G. D. (2015). sports specialization is associated with an increased risk of developing anterior knee pain in adolescent female athletes. Journal of sport rehabilitation, 24(1), 31-35. doi:: 10.1123/jsr.2013-0101

Harter, S. (1978). Effectance motivation reconsidered: Toward a developmental model. Human Development, 21(1), 3464. doi: $10.1159 / 000271574$

Helsen, W., Van-Winckel, J., \& Williams, A. (2005). The relative age effect in youth soccer across Europe. Journal of sports sciences, 23(6) 629-36. Doi:: 10.1080/

\section{0}

Helsen, W., Starkes, J., \& Hodges, N. (1998). Team sports and the theory of deliberate practice. Journal of Sport \& Exercise Psychology 20(1):12-34. Doi: 10.1123/ jsep.20.1.12

Hendry, D. T., Crocker, P. R. E., \& Hodges, N. J. (2014). Practice and play as determinants of self-determined motivation in youth soccer players. Journal of sports sciences, 32(11), 1091-1099. doi:: 10.1080/02640414.2014.880792

Henry I. Athlete Development, Athlete Rights and Athlete Welfare: A European Union Perspective. The International journal of the history of sport. 2013; 30(4): 356-373

Hill, G. M. (1993). Youth participation of professional baseball players. Sociology of Sport Journal, 10, 107-114. doi.org/ 10.1123/ssj.10.1.107

Hornig, M., Aust, F., \& Gullich, A. (2016). Practice and play in the development of German top-level professional football players. European journal of sport science, 16(1), 96-105. doi: 10.1080/17461391.2014.982204

International Federation of Sports Medicine. Excessive physical training in children and adolescents: a position statement. Sport Health 9:23-24, 1991.

Jayanthi, N., \& Dugas, L. (2017). The risks of sports specialization in the adolescent female athlete. Strength and Conditioning Journal, 39, 20-26. doi: 10.1519/ SSC.0000000000000293

Jayanthi, N., LaBella, C. R., Fischer, D., Pasulka, J., \& Dugas, L. R. (2015). Sports-specialized intensive training and the risk of injury in young athletes: a clinical case-control study. The American journal of sports medicine, 43(4), 794-801. doi: 10.1177/0363546514567298

Jayanthi, N., Pinkham, C., Dugas, L., Patrick, B., \& Labella, C. (2013). Sports specialization in young athletes: evidencebased recommendations. Sports Health, 5(3), 251-257. doi: $10.1177 / 1941738112464626$

Jayanthi, N., Dechert, A., Durazo, R., Dugas, L., \& Luke, A. (2011). Training and specialization risks in junior elite tennis players. Journal Of Medicine And Science In Tennis, 16, 14-20.

Johnson, M., Tenenbaum, G., \& Edmonds, W. (2006). Adaptation to physically and emotionally demanding conditions: the role of deliberate practice. High Ability Studies, 17(1), 117-136. doi: 10.1080/13598130600947184

Jones, B., Lawrence, G., \& Hardy, L. (2018). New evidence of relative age effects in «super-elite» sportsmen: a case for the survival and evolution of the fittest. Journal of sports sciences, 36(6), 697-703. doi: 10.1080/ 02640414.2017.1332420

Kalinowski, A. G. (1985). The development of Olympic swimmers. In B. S. Bloom (Ed.), Developing talent in young people (pp. 139-192).New York:Ballantine Books.

Knapik, D.,Rizzone, K., \& Voos, J. (2020) Timing and Reasons Behind Single-Sport

Specialization in Soccer: A Survey of 64 Major League Soccer Athletes. Sports Health. 12(4)355-360.

Krogius, N. (1976). Psychology in chess. New York: RHM Press.

LaPrade, R., Agel, J., Baker, J., Brenner, J., Cordasco, F., Cote, J.,Provencher, M. (2016). AOSSM Early sport 
specialization consensus statement. Orthop J Sports Med, 4(4), 2325967116644241. doi: 10.1177/ 2325967116644241

Latorre, P., Garcia, F., \& López, J. (2018) Early sport dropout: High performance in early years in young athletes is not related with later success. Retos, 33.210-2012

Law M, Stewart D, Pollock N. (1998.). Critical review form: quantitative studies. Hamilton: MacMaster University.

Law, M., Côté, J., \& Anders Ericsson, K. (2008). Characteristics of expert development in rhythmic gymnastics: A retrospective study. International. Journal of Sport and Exercise Psychology, 5(1) 82-103doi10.1080/ 1612197X.2008.9671814.

Le Gall, F., Carling, C., Williams, M., \& Reilly, T. (2010). Anthropometric and fitness characteristics of international, professional and amateur male graduate soccer players from an elite youth academy. Journal of science and medicine in sport, 13(1), 90-95. doi: 10.1016/ j.jsams.2008.07.004

Leite, N., \& Sampaio, J. (2012). Long-term athletic development across different age groups and gender from Portuguese basketball players. International Journal of Sports Science and Coaching, 7, 285-300. doi: 10.1260/17479541.7.2.285

Lemez, S., MacMahon, C., \& Weir, P. (2016). Relative age effects in women's rugby union from developmental leagues to world cup tournaments. Research quarterly for exercise and sport, 87(1), 59-67. doi: 10.1080/ 02701367.2015.1116120

Letts L, Wilkins S, Stewart D, Bosch, J., \& Westmorland, M. (2007). Critical review form: qualitative studies (version 2.0). Hamilton: MacMaster University.

Lopez de Subijana, C., \& Lorenzo, J. (2018). Relative age effect and long-term success in the spanish soccer and basketball national teams. Journal of human kinetics, 65, 197-204. doi: 10.2478/hukin-2018-0027

MacMahon, C., Helsen, W., Starkes, J., \& Weston, M. (2007). Decision-making skills and deliberate practice in elite association football referees. Journal of sports sciences, 25(1), 65-78. doi: 10.1080/02640410600718640

Macnamara, B., Moreau, D., \& Hambrick, D. (2016) The Relationship Between Deliberate Practice and Performance in Sports. Perspectives on Psychological Science 11,33-350.

Malina, R. (2010). Early sport specialization: roots, effectiveness, risks. Curr Sports Med Rep, 9(6), 364-371. doi: 10.1249/JSR.0b013e3181fe3166

Martin, R. P., Foels, P., Clanton, G., \& Moon, K. (2004). Season of birth is related to child retention rates, achievement, and rate of diagnosis of specific LD. Journal of learning disabilities, 37(4), 307-317. doi: 10.1177/ 00222194040370040301

Martindale, R., Collins, D., \& Daubney, J. (2005). Talent development: A guide for practice and research within sport. Quest, 57(4), 353-375. doi: 10.1080/ 00336297.2005 .10491862

McCarthy, N., Collins, D., \& Court, D. (2016). Start hard, finish better: further evidence for the reversal of the RAE advantage. Journal of sports sciences, 34(15), 1461-1465. doi: 10.1080/02640414.2015.1119297
Memmert, D., Baker, J., \& Bertsch, C. (2010). Play and practice in the development of sport-specific creativity in team ball sports. High Ability Studies, 21, 3-18. doi:10.1080/ 13598139.2010.488083

Meister, K., Day, T., Horodyski, M., Kaminski, T., Wasik, M., \& Tillman, S. (2005). Rotational motion changes in the glenohumeral joint of the adolescent/Little League baseball player. The American journal of sports medicine, 33(5), 693-698. doi: 10.1177/0363546504269936

Moesch, K., Trier Hauge, M.-L., Wikman, J., \& Elbe, A.-M. (2013). Making it to the top in team sports: start later, intensify, and be determined!. Talent Development and Excellence 5(2):85-100

Moesch, K., Elbe, A., Hauge, M., \& Wikman, J. M. (2011). Late specialization: the key to success in centimeters, grams, or seconds (cgs) sports. Scandinavian journal of medicine \& science in sports, 21(6), e282-290. doi: 10.1111/j.1600-0838.2010.01280.x

Moher, D., ShamsERE, L., Clarke, M., Ghersi, D., Liberati,A., \& Petticrew, M (2015). Preferred reporting items for systematic review and meta-analysis protocols (PRISMA-P) 2015 statement. Systematic Reviews, 4(1), 1. doi: $10.1186 / 2046-4053-4-1$

Monsaas, J. A. (1985). Learning to be a world-class tennis player. In B. S. Bloom (Ed.), Developing talent in young people (pp. 211 -269). New York: Ballantine Books

Mostafavifar, A., Best, T., \& Myer, G. (2013). Early sport specialisation, does it lead to long-term problems? British journal of sports medicine, 47(17), 1060-1061. doi: 10.1136/ bjsports-2012-092005

Müller, L., Hildebrandt, C., \& Raschner, C. (2015). The relative age effect and the influence on performance in youth alpine ski racing. Journal of Sports Science \& Medicine, 14(1), 16-22.

Musch, J., \& Grondin, S. (2001). Unequal competition as an impediment to personal development: A review of the relative age effect in sport. Developmental Review, 21(2), 147-167. doi: 10.1006/drev.2000.0516

Myer, G., Jayanthi, N., Difiori, J., Faigenbaum, A., Kiefer, A., Logerstedt, D., \& Micheli, L. (2015). Sport specialization, part i: does early sports specialization increase negative outcomes and reduce the opportunity for success in young athletes? Sports Health, 7(5), 437-442. doi: 10.1177/ 1941738115598747

Myer, G., Jayanthi, N., DiFiori, J., Faigenbaum, A., Kiefer, A., Logerstedt, D., \& Micheli, L.(2016). Sports specialization, part II: Alternative solutions to early sport specialization in youth athletes. Sports Health, 8(1), 65-73. doi: 10.1177/ 1941738115614811

Nakata, H. (2017a). Relationship between the relative age effect and lengths of professional carEREs in male japanese baseball players: A retrospective analysis. Sports Medicine - Open, 3(1), 21-21. doi: 10.1186/s40798017-0090-3

Nakata, H. (2017b). Relationship between the relative age effect and lengths of professional carEREs in male japanese baseball players: A retrospective analysis. Sports Medicine - Open, 3, 21. doi: 10.1186/s40798-0170090-3

Nakata, H., \& Sakamoto, K. (2011). Relative age effect in 
Japanese male athletes. Perceptual and motor skills, 113(2), 570-574. doi: 10.2466/05.10.11.pms.113.5.570-574

Pankhurst, A., Collins, D., \& Macnamara, A. (2013). Talent development: linking the stakeholders to the process. Journal of sports sciences, 31(4), 370-380. doi: 10.1080/ 02640414.2012.733821

Post, E., Trigsted, S., Riekena, J., Hetzel, S., McGuine, T., Brooks, M., \& Bell, D. (2017). The association of sport specialization and training volume with injury history in youth athletes. The American journal of sports medicine, 45(6), 1405-1412. doi: 10.1177/0363546517690848

Rea, T., \& Lavallee, D. (2015). An examination of athletes' experiences of the talent transfer process. Talent Development \& Excellence, 7 (1) 41-67

Rees, T., Hardy, L., Gullich, A., Abernethy, B., Cote, J., Woodman, T., Warr, C. (2016). The great british medalists project: a review of current knowledge on the development of the world's best sporting talent. Sports medicine, 46(8), 1041-1058. doi: 10.1007/s40279-016-0476-2

Romann, M., \& Cobley, S. (2015). Relative age effects in athletic sprinting and corrective adjustments as a solution for their removal.PLoS One,10 (4) 1-12 10.1371/ journal.pone.0122988

Ronbeck, N., Dunnagan, T., \& Stewart, C. (2005). Early specialization in elite Nordic racers: Fact or fiction. Retrieved April 13, 2010, from www.coachesinfo.com/

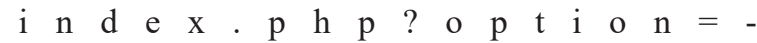
com_content\&view $=$ article $\& i d=315$ :better-coach nordic\&catid $=91$ :general-articles\&itemid $=170$

Rowland, T.W. Counseling the young athlete: where do we draw the line? Ped. Exec Sci. 9:197-201, 1997

Rubajczyk, K., Ewierzko, K.,\& Rokita, A. (2017) Doubly Disadvantaged? The Relative

Age Effect in Poland's Basketball Players Journal of sports science \& medicine, 16(2), 280-285.

Rubia, A., Bjørndal, C., Sanchez, J., Yague, J., Calvo, J., \&Maroto, S. (2020) The relationship between the relative age effect and performance among athletes in World Handball Championships. PLoS ONE 15(3): e0230133. https://doi.org/10.1371/journal.pone.0230133

Sarmento, H., Anguera, M., Pereira, A., \& Araujo, D. (2018). Talent Identification and Development in Male Football: A Systematic Review. Sports medicine, 48(4), 907-931. doi: 10.1007/s40279-017-0851-7

Seiler, S., \& Tonnessen, E. (2009). Intervals, thresholds, and long slow distance: The role of intensity and duration in endurance training. Sport science, 13, 32-53. Retrieved from sportsci.org/ 2009/ss.htm

Simon, H., \& Chase, W.(1973). Skill in chess. American Scientist, 61(4), 394-403.

Smith, R. (1986). Toward a cognitive-affective model of athletic burnout. Journal of Sport Psychology, 8(1), 36-50. doi: 10.1123/jsp.8.1.36

Soberlak, P., \& Cote, J. (2003). The developmental activities of elite ice hockey players. Journal of Applied Sport Psychology, 15(1), 41-49. doi: 10.1080/10413200305401

Sosniak, L. A. (1985). Learning to be a concert pianist. In B. S. Bloom (Ed.), Developing talent in young people (pp. 1967). New York: Ballantine Books.

Starkes, J. (2000). The road to expertise: is practice the only determinant?

International journal of sport psychology, 31(4):431-451

Timothy, A., Eric, G., Scott, J., Brooks, M., Stephanie, T., \& David, R. (2017). A prospective study on the effect of sport specialization on lower extremity injury rates in high school athletes. The American journal of sports medicine, 45(12), 2706-2712. doi: 10.1177/0363546517710213

Vaeyens, R., Gullich, A., Warr, C., \& Philippaerts, R. (2009). Talent identification and promotion programmes of Olympic athletes. Journal of sports sciences, 27(13), 13671380. doi: 10.1080/02640410903110974

Vaeyens, R., Lenoir, M., Williams, A. M., \& Philippaerts, R. M. (2008). Talent identification and development programmes in sport : current models and future directions. Sports medicine, 38(9), 703-714.

Van Rossum, J. (2000). Deliberate practice and Dutch field hockey: An addendum to Starkes. International Journal of Sport Psychology, 31, 452-460.

Wall, M., \& Côté, J. (2007). Developmental activities that lead to dropout and investment in sport. J Appl Sport Psychol, 20. doi: 10.1080/17408980601060358

Wallingford, R. (1975). Long distance running. In A. W Tayler \& F Landry (Eds.), The scientific aspects of sports training (pp. 118-130). Springfield, IL: Charles C Thomas.

Ward, P., Hodges, N., Williams, M., \& Starkes, J. (2007). The road to excellence in soccer: A quasi-longitudinal approach to deliberate practice.High Ability Studies, 18(2), 119-153.

Weissensteiner, J., Abernethy, B., Farrow, D., \& Muller, S. (2008). The development of anticipation: a cross-sectional examination of the practice experiences contributing to skill in cricket batting. Journal of sport \& exercise psychology, 30(6), 663-684.

Wierike, S., Van der Sluis, A., Van den Akker-Scheek, I., Elferink-Gemser, M., \& Visscher, C. (2013). Psychosocial factors influencing the recovery of athletes with anterior cruciate ligament injury: a systematic review. Scandinavian journal of medicine \& science in sports, 23(5), 527-540. doi: 10.1111/sms.12010

Wolpert, D., Diedrichsen, J., \& Flanagan, J. (2011). Principles of sensorimotor learning. Nature reviews. Neuroscience, 12(12), 739-751. doi: $10.1038 / \mathrm{nrn} 3112$

World Health Organization and International Federation of Sports Medicine (1997). Sports and children. Olympic Coach 7(3):6-8.

Wright, A., \& Côté, J. (2003). A retrospective analysis of leadership development through sport. Sport Psychologist,17(3)268-291.doi: 10.1123/tsp.17.3.268

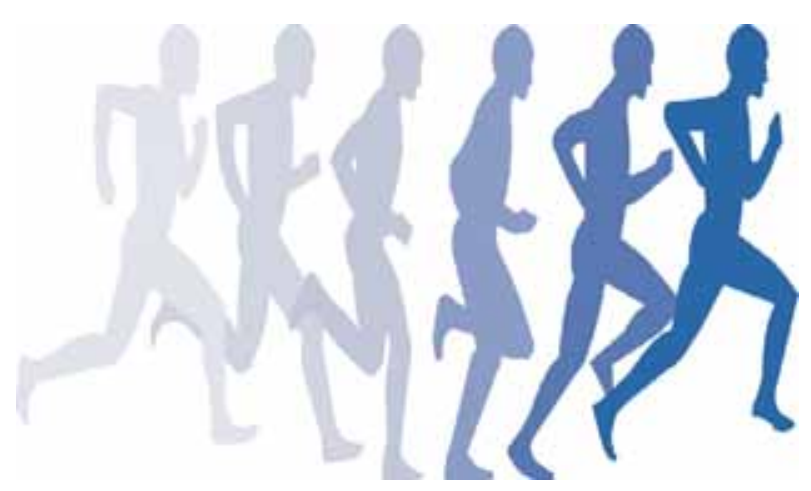

